\title{
Jan Seidler
}

Ergodic behaviour of stochastic parabolic equations

Czechoslovak Mathematical Journal, Vol. 47 (1997), No. 2, 277-316

Persistent URL: http://dml.cz/dmlcz/127357

\section{Terms of use:}

(C) Institute of Mathematics AS CR, 1997

Institute of Mathematics of the Czech Academy of Sciences provides access to digitized documents strictly for personal use. Each copy of any part of this document must contain these Terms of use.

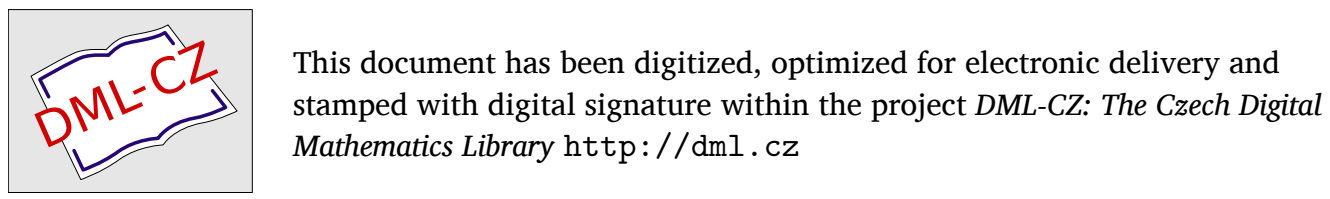




\title{
ERGODIC BEHAVIOUR OF STOCHASTIC PARABOLIC EQUATIONS*
}

\author{
JAN SEIDLER, Praha
}

(Received November 28, 1995)

Dedicated to Professor Jaroslav Kurzweil on the occasion of his $70^{\text {th }}$ birthday

Summary. The ergodic behaviour of homogeneous strong Feller irreducible Markov processes in Banach spaces is studied; in particular, existence and uniqueness of finite and $\sigma$-finite invariant measures are considered. The results obtained are applied to solutions of stochastic parabolic equations. tions

Keywords: Markov processes, invariant measures, recurrence, stochastic parabolic equa-

MSC 1991: 60H15, 60J35

\section{INTRODUCTION}

This paper is devoted to the study of the asymptotic behaviour of Markov processes arising as solutions to the semilinear stochastic differential equation of the type

$$
\mathrm{d} X=(A X+F(X)) \mathrm{d} t+\Sigma(X) \mathrm{d} W
$$

in a Hilbert space $H$. There are many results on the existence, uniqueness and stability of invariant measures for (1.1) (see e.g. the monograph [7] and references therein), nevertheless, if $H$ is finite dimensional a more complete description is available, provided the noise is sufficiently nondegenerated. To be more precise, let us consider a stochastic differential equation

$$
\mathrm{d} X=b(X) \mathrm{d} t+\sigma(X) \mathrm{d} w
$$

\footnotetext{
* This research has been partially supported by the AVCR Grant No. 11965.
} 
in $\mathbb{R}^{n}$ where $w$ is a standard Wiener process in $\mathbb{R}^{n}$ and the coefficients $b, \sigma$ are, say, Lipschitz continuous. If the diffusion matrix $\sigma \sigma^{*}$ is (strictly) positive everywhere in $\mathbb{R}^{n}$ then the Markov process $X$ solving (1.2) is strong Feller and irreducible (cf. [21], Chapter IV, [40] and [41], or [4]). In the late fifties G. Maruyama and H. Tanaka ([24], [25]), and independently R. Khas'minskiǔ ([20]) developed the embedded Markov chain technique which made it possible to investigate in a very lucid way the long time behaviour of strong Feller irreducible Markov processes in locally compact spaces; see also the papers [1], [2], [9], [29], [30] for further development of the theory. It turns out that certain dichotomy holds: Either there exists an invariant probability for $X$, then the process $X$ is Harris recurrent, the invariant measure is unique and the transition function converges to it in the total variation norm. Or there is no invariant probability, then the transition function approaches the unit point mass at infinity in the weak* topology of the space of signed measures on $\mathbb{R}^{n}$. Moreover, the existence of an invariant measure is closely related to the existence of a recurrent compact subset of the state space.

A naïve attempt to extend these results to infinite-dimensional Hilbert spaces in a straightforward way fails. B. Maslowski considered a linear stochastic evolution equation of the form

$$
\mathrm{d} X=A X \mathrm{~d} t+Q \mathrm{~d} W
$$

in a Hilbert space $K=L^{2}((0, \infty))$, where $W$ is a standard cylindrical Wiener process in $K, A$ is an infinitesimal generator of a strongly continuous semigroup on $K, Q$ is a Hilbert-Schmidt operator in $K$ (so the mild solutions of (1.3) are well defined) such that $Q>0$. He showed that $A$ and $Q$ can be chosen such that the equation (1.3) admits infinitely many invariant probabilities, albeit $Q$ is strictly positive. (See [27], Example 3.8; the idea of the example goes back to the paper [42], where nonuniqueness was established but with $Q$ only nonnegative.) It is important, however, to note that the solution of (1.3) is irreducible but lacks the strong Feller property. In the last few years (or even months) there has been a considerable progress in establishing that solutions to particular stochastic parabolic problems are strong Feller irreducible Markov processes (see e.g. [26], [33], [5], [6], [12]). So it seems to be of some interest to investigate whether we can obtain for these solutions results analogous to the dichotomy mentioned above. The aim of this paper is to show that it is so and that the results established under the local compactness assumption remain, to a great extent, valid in a more general situation. An attempt in this direction was undertaken in [28] where semilinear parabolic equations with an additive noise were investigated, the construction of the embedded chain relying heavily on the special structure of the considered equations (cf. also [34] for a related construction in the 
case of stochastic delay equations). In this paper we work directly with strong Feller irreducible homogeneous Markov processes in separable Banach spaces (or, more generally, in Polish spaces), so, in particular, we can cover a wider class of stochastic equations.

The paper is organized as follows. In the next section we present the main results on the dichotomy, which are in Section 3 applied to stochastic parabolic problems. The fourth section contains the proof of Theorem 2.1 concerning the transient case, whilst the last section is devoted to proofs of the results on the recurrent case. Some propositions established here may be of independent interest. We carry out two different constructions of a $\sigma$-finite invariant measure. The first is based on the classical procedure from [25] or [20]: assuming the existence of a recurrent compact set $K$ we construct a Feller Markov chain living in $K$ the invariant measure of which yields the invariant measure of the whole process in a standard manner; this approch gives us immediately criteria for the positive recurrence (that is, the finiteness of the invariant measure). The other construction employs Foguel's theorem on the existence of a $\sigma$-finite subinvariant measure and works not only if there is a recurrent compact set but also under the supposition that-roughly speaking-all open sets are recurrent, which is of some importance in applications to stochastic evolution equations. In the course of the proofs we establish the Harris recurrence of the process considered but to do so we need a fairly detailed knowledge of the invariant measure and its properties, thus we will not use the abstract Harris result on the existence of a $\sigma$-finite invariant measure.

Acknowledgement. I wish to express my thanks to B. Maslowski for many useful discussions on the topic of the paper. A. Chojnowska-Michalik offered valuable comments concerning Example 3.4.

\section{MAin RESUlts}

Let $H$ be a separable Banach space with the norm $\|\cdot\|$. Let us denote by $\mathscr{B}(H)$ the Borel sets in $H$, by $\mathbf{B}(H)$ the space of all bounded Borel functions on $H$ equipped with the norm $\|f\|_{\infty}=\sup _{H}|f|, f \in \mathbf{B}(H)$, and by $\mathscr{C}_{b}(H)$ the space of all bounded continuous functions on $H$ with the same norm. The norm of the dual space $\mathbf{B}(H)^{*}$ will be denoted by $\|\cdot\|$. In particular, if $\nu$ is a Borel signed measure on $H$ then $\|\nu\|$ coincides with the total variation of $\nu$. The symbol $\chi_{C}$ will stand for the characteristic function of a set $C$. Everywhere in the sequel, we will use the convention $\inf \emptyset=+\infty$.

Assume that $X=\left(\Omega, \mathscr{F},\left(\mathscr{F}_{t}\right),\left(X_{t}\right), \mathrm{P}_{x}\right)$ is a Markov process in $H$ with a transition function $P$ such that $P(s, x, t, A)=P(0, x, t-s, A) \equiv P(t-s, x, A)$. Then there 
exists an equivalent process which is homogeneous in the sense of [10], $\$ 2.2$ (see [10], Theorem 2.10), let us assiume (without any loss of generality) that $X$ is just this homogeneous process. Let $\left(\theta_{t}\right)$ be the corresponding shift operators. When convenient, we will write $X(s)$ instead of $X_{s}$. Let us define

$$
P_{t}: \mathbf{B}(H) \longrightarrow \mathbf{B}(H), \quad P_{t} f(\cdot)=\int_{H} f(y) P(t, \cdot, \mathrm{d} y), \quad t>0 .
$$

Denote by $P_{t}^{*}, t>0$, the dual operators on $\mathbf{B}(H)^{*}$, in particular, for any finite Borel measure $\nu$ on $H$ one has

$$
P_{t}^{*} \nu(\cdot)=\int_{H} P(t, x, \cdot) \mathrm{d} \nu(x)
$$

This formula makes it possible to define $P_{t}^{*} \nu$ for any (nomnegative, but not necessarily finite) measure $\nu$ on $\mathscr{B}(H)$. We say that a measure $\mu \neq 0$ is invariant for the process $X$ if $P_{t}^{*} \mu=\mu$ for all $t>0$.

Let us adopt the following assumptions:

(A1) The process $X$ has continuous sample paths.

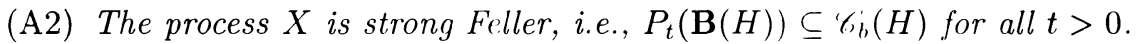

(A3) The process $X$ is irreducible, i.e., $P(t, x, U)>0$ for all $t>0, x \in H$, and $U \neq \emptyset$ open sets in $H$.

Note that due to (A1) and (A2) the process $X$ is strong Markov (see [10], Theorem 5.10). Furthermore, (A2) and (A3) imply that all the measures $P(t, x, \cdot), t>0$. $x \in H$, are equivalent (see [20], $7^{\circ}$ ). Let us fix an arbitrary one of them and denote it by $\varphi$, for definiteness, we sot $\varphi=P(1,0, \cdot)$. If there exists an invariant measure $\mu$ for $X$ then $\mu$ and $\varphi$ are equivalent as well. Finally, (A1) yields that the function $(t, x) \longmapsto P(t, x, A)$ is $\mathscr{B}((0, \infty)) \bigcirc \mathscr{B}(H)$-measurable for every $A \in \mathscr{B}(H)$ (cf. [10]. Remark following Lemma 5.3).

Up to now, our assumptions corresponded to the (lassical ones. The last assump)tion

(A4) $\forall K \subseteq H$ a compact set $\forall \eta>0$

$$
\lim _{T \rightarrow 0+} \sup _{y \in K} \mathrm{P}_{y}\left\{\sup _{0 \leqslant t \leqslant T}\left\|X_{t}-y\right\| \geqslant \eta\right\}=0
$$

is stronger than its counterpart in the locally compar't case and may be a bit unnatural outside the framework of applications to solutions of stochastic evolution equations.

Definition 2.1. Let $A \in \mathscr{B}(H)$ be a Borel set. Denoting by

$$
\tau_{A}=\inf \left\{t>0 ; X_{t} \in A\right\}
$$


the first hitting time of $A$, we say that the set $A$ is recurrent (for the process $X$ ) provided $\mathrm{P}_{x}\left\{\tau_{A}<\infty\right\}=1$ for all $x \in H$.

Recall that $\tau_{A}$ is a stopping time whenever $X$ has continuous paths and $A$ is closed. If the filtration $\left(\mathscr{F}_{t}\right)$ fulfils the usual conditions then $\tau_{A}$ is a stopping time for every Borel set $A$, see e.g. [8], Theorem IV.50.

Now we are prepared to state the first theorem.

Theorem 2.1. Let the process $X$ fulfil the assumptions (A1)-(A4). Let $K \subseteq H$ be a compact set which is not recurrent, then

$$
\sup _{x \in H} \int_{0}^{\infty} P(t, x, K) \mathrm{d} t<\infty
$$

and $\mu(K)=0$ for any finite invariant measure $\mu$. If, moreover, $\varphi(K)>0$ then

$$
\mathrm{P}_{x}\left\{\sup \left\{t \geqslant 0 ; X_{t} \in L\right\}<\infty\right\}=1
$$

and

$$
\lim _{t \rightarrow \infty} P(t, x, L)=0
$$

hold for all $x \in H$ and any compact set $L$ in $H$.

In other words, if there exists a finite invariant measure for $X$ then one can find a recurrent compact set in $H$, since finite Borel measures on $H$ are Radon.

Before we state the next theorem recall that a family $\left(\alpha_{t}, t \geqslant 0\right)$ of real random variables on $\Omega$ is called an additive functional provided $\alpha_{t}$ is $\mathscr{F}_{t}$-measurable for any $t \geqslant 0$ and $\alpha_{s+t}=\alpha_{s}+\theta_{s} \alpha_{t}$ holds $\mathrm{P}_{x}$-almost surely for any $x \in H, s, t \geqslant 0$. We say that $\left(\alpha_{t}\right)$ is nonnegative if $\mathrm{P}_{x}\left\{\alpha_{t} \geqslant 0\right\}=1$ for all $x$ and $t \geqslant 0$. For any measure $\nu$ on $\mathscr{B}(H)$ define a measure $\mathrm{P}_{\nu}$ on $\mathscr{F}$ by

$$
\mathrm{P}_{\nu}(B)=\int_{H} \mathrm{P}_{x}(B) \mathrm{d} \nu(x), \quad B \in \mathscr{F},
$$

let $E_{\nu}$ denote the integral with respect to $P_{\nu}$. Finally, recall that we say that a measure $\varrho$ is Radon provided $\varrho$ is locally finite and compact inner regular, i.e. each point has an open neighbourhood $V$ with $\varrho(V)<\infty$, and $\varrho(B)=\sup \{\varrho(K) ; K \subseteq$ $B, K$ compact $\}, B \in \mathscr{B}(H)$.

Theorem 2.2. Let the assumptions (A1)-(A4) be satisfied. Assume that there exists a recurrent compact set $\Lambda \subseteq H$. Then:

(i) There exists a $\sigma$-finite invariant measure $\mu$ for the process $X$. Up to a multiplicative constant, $\mu$ is the unique $\sigma$-finite invariant measure. Moreover, $\mu$ is Radon. 
(ii) For $\eta>0$ denote by $\mathfrak{n}_{\eta}$ the first hitting time of $K$ after $\eta$, that is

$$
\mathfrak{n}_{\eta}=\inf \left\{t>\eta ; X_{t} \in K^{\prime}\right\}
$$

Assume that there exists $\delta>0$ such that

$$
\sup _{x \in K} \mathrm{E}_{x} \mathfrak{n}_{\delta}<\infty
$$

Then $\mu(H)<\infty$.

(iii) The process $X$ is Harris recurrent, that is

$$
\mathrm{P}_{x}\left\{\int_{0}^{\infty} \chi_{A}\left(X_{s}\right) \mathrm{d} s=+\infty\right\}=1
$$

holds for any $x \in H$ and all $A \in \mathscr{B}(H)$ such that $\mu(A)>0$.

(iv) Let $\left(A_{t}\right),\left(B_{t}\right)$ be additive functionals, $\left(B_{t}\right)$ nonnegative, and suppose that $\mathrm{E}_{\mu}\left|A_{1}\right|<\infty, 0<\mathrm{E}_{\mu} B_{1}<\infty$. Then

$$
\mathrm{P}_{x}\left\{\lim _{t \rightarrow \infty} \frac{A_{t}}{B_{t}}=\frac{\mathrm{E}_{\mu} A_{1}}{\mathrm{E}_{\mu} B_{1}}\right\}=1
$$

holds for all $x \in H$. Moreover,

$$
\lim _{t \rightarrow \infty} \frac{\mathrm{E}_{x} A_{t}}{\mathrm{E}_{x} B_{t}}=\frac{\mathrm{E}_{\mu} A_{1}}{\mathrm{E}_{\mu} B_{1}}
$$

holds for $\mu$-almost every $x \in H$.

(v) For any probability measures $\pi$, $\varrho$ on $\mathscr{B}(H)$ one has

$$
\lim _{t \rightarrow \infty}\left\|P_{t}^{*} \pi-P_{t}^{*} \varrho\right\|=0
$$

The theorem has many immediate consequences, some of them we state explicitly because of their importance.

Corollary 2.3. Let the assumptions of Theorem 2.2 be fulfilled, let $\mu$ be the $\sigma$-finite invariant measure for $X$.

a) Let $\mu(H)=\infty$. Then

$$
\lim _{T \rightarrow \infty} \frac{1}{T} \int_{0}^{T} \beta\left(X_{s}\right) \mathrm{d} s=0 \quad \mathrm{P}_{x} \text {-almost surely }
$$


and

$$
\lim _{t \rightarrow \infty} P_{t} f(x)=0
$$

for any $\beta \in L^{1}(\mu), f \in L^{1}(\mu) \cap \mathbf{B}(H)$ and all $x \in H$. In particular,

$$
\lim _{t \rightarrow \infty} P(t, x, D)=0
$$

whenever $x \in H$ and $D \in \mathscr{B}(H)$ satisfies $\mu(D)<\infty$.

b) Let $\mu(H)<\infty$. Set $\mu^{*}(\cdot)=\mu(\cdot) / \mu(H)$ then $\mu^{*}$ is an invariant probability measure and

$$
\lim _{T \rightarrow \infty} \frac{1}{T} \int_{0}^{T} \psi\left(X_{s}\right) \mathrm{d} s=\int_{H} \psi \mathrm{d} \mu^{*} \quad \mathrm{P}_{x} \text {-almost surely }
$$

for any $x \in H$ and $\psi \in L^{1}(\mu)$. Further,

$$
\lim _{t \rightarrow \infty}\left\|P_{t}^{*} \pi-\mu^{*}\right\|=0
$$

for every probability measure $\pi$ on $\mathscr{B}(H)$. In particular,

$$
\lim _{t \rightarrow \infty}\left\|P(t, x, \cdot)-\mu^{*}\right\|=0
$$

for any $x \in H$.

Remark 2.1. a) For us, the most important particular choice in (2.3) and (2.4) is

$$
A_{t}=\int_{0}^{t} f\left(X_{s}\right) \mathrm{d} s, \quad B_{t}=\int_{0}^{t} g\left(X_{s}\right) \mathrm{d} s,
$$

where $f, g \in L^{1}(\mu), g \geqslant 0$ and $\int_{H} g \mathrm{~d} \mu>0$. Then we get

$$
\mathrm{P}_{x}\left\{\lim _{t \rightarrow \infty} \frac{\int_{0}^{t} f\left(X_{s}\right) \mathrm{d} s}{\int_{0}^{t} g\left(X_{s}\right) \mathrm{d} s}=\frac{\int_{H} f \mathrm{~d} \mu}{\int_{H} g \mathrm{~d} \mu}\right\}=1
$$

for any $x \in H$, and

$$
\lim _{t \rightarrow \infty} \frac{\int_{0}^{t} P_{s} f(x) \mathrm{d} s}{\int_{0}^{t} P_{s} g(x) \mathrm{d} s}=\frac{\int_{H} f \mathrm{~d} \mu}{\int_{H} g \mathrm{~d} \mu}
$$

for $\mu$-almost all $x \in H$. 
b) With the choice $\pi=\delta_{x}, \varrho=\delta_{y}$ the formula (2.5) yields

$$
\lim _{t \rightarrow \infty}\|P(t, x, \cdot)-P(t, y, \cdot)\|=0
$$

for all $x, y \in H$, thus also

$$
\lim _{t \rightarrow \infty}\left|P_{t} f(x)-P_{t} f(y)\right|=0
$$

for all $x, y \in H$ and $f \in \mathbf{B}(H)$.

c) Note that (2.7) yields

$$
\lim _{t \rightarrow \infty} P_{t} f(x)=\int_{H} f \mathrm{l} \mu^{*}
$$

for all $f \in \mathbf{B}(H)$ and $x \in H$. Further, (2.6) can be restated as

$$
\lim _{t \rightarrow \infty} f\left(X_{t}\right)=0 \quad \text { in } \quad L^{1}\left(\mathrm{P}_{. l}\right)
$$

for any $x \in H$ and all $f \in L^{1}(\mu) \cap \mathbf{B}(H)$.

Remark 2.2. The sufficient condition (2.2) for the finiteness of $\mu$ (proposed in [29], Theorem 1.2) is simple, but sometimes a morlified version introduced by Khas'minskiŭ (see [20], Theorem 3.3, or [21], §IV.4) may be more useful. Let $V \subseteq H$ be a bounded open set, $V \supseteq K$. and let $\mathfrak{p}$ be the first hitting time of $K$ after hitting $H \backslash V$, i.e. if $\sigma$ is the first hitting time of $H \backslash V$ and $\tau$ is the first hitting time of $\Lambda$ then $\mathfrak{p}$ is defined by $\mathfrak{p}=\sigma+\theta_{\sigma} \tau$. Again, if

$$
\sup _{x \in K} E_{x} \mathfrak{p}<\infty
$$

then $\mu(H)<\infty$. As shown in $[21]$, in the case $H=\mathbb{R}^{\prime \prime}$ either of the conditions

$$
\sup _{x \in K} \mathrm{E}_{l} \sigma<\infty, \quad \sup _{x \in \partial V} \mathrm{E}_{x} \tau<\infty
$$

implies the other and hence also (2.8); I do not know whether something like this remains true in our setting.

The existence of a recurrent compact set has far-reaching consequences. It seems difficult, however, to verify directly that some compact set is recurrent for a solution of a stochastic evolution equation. On the other hand, the Lyapunov function criteria for recurrence (see e.g. [21], Theorem III.7.1) extend, at least in principle, to infinite-dimensional diffusions, but they yield recurrence of balls. Tracing the proof 
of Theorem 2.2 one can notice that the argument remains valid if we assume only that there are sufficiently many recurrent balls. We will need a new hypothesis, namely

(A5) $\forall x \in H \quad \forall \varepsilon, \eta>0 \quad \exists V$ an open neighbourhood of $x \quad \exists T>0$

$$
\sup _{y \in V} P_{y}\left\{\sup _{0 \leqslant t \leqslant T}\left\|X_{t}-y\right\| \geqslant \eta\right\} \leqslant \varepsilon
$$

Note that (A5) is a strengthened form of the assumption (A4), nonetheless it is still fulfilled in applications we have in mind.

Proposition 2.4. Let the assumptions (A1)-(A3) and (A5) be fulfilled. Suppose that a point $x_{0} \in H$ can be found such that there exists a local base at $x_{0}$ consisting of recurrent neighbourhoods. Then there exists a recurrent compact set $K$ in $H$.

In connection with Proposition 2.4 let us mention that the relation between the existence of an invariant measure for a linear stochastic evolution equation in a Hilbert space and the recurrence of open sets was investigated already in the paper [42], but in a context substantially different from ours.

Many steps of the proof of Theorem 2.2 do not require the full strength of the assumptions (A2), (A3), but only their easy consequence, the equivalence of transition probabilities. In fact, our proof yields also the following proposition (virtually well known, see e.g. [39], Theorem 1, for a different approach), showing that we can indeed relax the assumptions provided we know a priori that there exists a finite invariant measure.

Proposition 2.5. Let the assumption (A1) be satisfied, and let all measures $P(t, x, \cdot), t>0, x \in H$, be equivalent. If there exists a finite invariant measure for the process $X$, then $X$ is Harris recurrent. In particular, the assertions (iv) and (v) of Theorem 2.2 hold.

Remark 2.3. We have stated the results in the generality that we need in applications to stochastic evolution equations. It can be observed easily that the linear structure of the space $H$ is never used, so both the results and the proofs remain valid whenever $H$ is an arbitrary Polish space (or, more generally, a separable metrizable Radon space). Further, the assumption (A3) can be omitted provided the transition probabilities are equivalent (see Remark 4.2 and Section 5 for precise formulations). 


\section{Applications to stochastic parabolic equations}

In this section we discuss several examples of particular stochastic parabolic equations to which the general theory developed in the previous section can be applied.

Example 3.1. Let us consider a stochastic evolution equation

$$
\mathrm{d} X=(A X+f(X)) \mathrm{d} t+b(X) \mathrm{d} W
$$

in a separable Hilbert space $H$, where $A: \operatorname{Dom}(A) \subseteq H \longrightarrow H$ is an infinitesimal generator of a $C_{0}$-semigroup $S(t)$ on $H$ and $W$ is a standard cylindrical Wiener process in $H$. Let us denote by $\mathscr{L}(H)$ the space of all bounded linear operators in $H$ and by $\|\cdot\|_{\text {HS }}$ the Hilbert-Schmidt norm of an operator. Assume that

(i) $f: H \longrightarrow H$ and $b: H \longrightarrow \mathscr{L}(H)$ are globally Lipschitz continuous on $H$,

(ii) the operators $b(z), z \in H$, are invertible and

$$
\sup _{z \in H}\left\|b(z)^{-1}\right\|<\infty,
$$

(iii) there exists $r \in] 0,1[$ such that

$$
\int_{0}^{T} t^{-r}\|S(t)\|_{\mathrm{HS}}^{2} \mathrm{~d} t<\infty
$$

for every $T>0$.

For $y \in H$ let $X(\cdot, y)$ stand for the mild solution of $(3.1)$ fulfilling $X(0, y)=y$. As is well known, the equation (3.1) defines a Markov process $X$ with the transition function $P(t, y, A)=\mathrm{P}\{X(t, y) \in A\}$. By [33] (Corollary 1.1 and Theorem 1.3) the process $X$ satisfies the assumptions (A2) and (A3). Moreover, (3.2) yields the continuity of paths of $X$, see [ 7 , Theorem 7.6. It remains to prove (A5). Fix $p>2 / r$ then there exists a constant $C_{1}<\infty$ such that

$$
\sup _{0 \leqslant t \leqslant 1} \mathrm{E}\|X(t, y)\|^{p} \leqslant C_{1}\left(1+\|y\|^{\prime}\right), \quad y \in H
$$

(see again Theorem 7.6 in [7]). Further, Proposition 7.9 from [7] yields

$$
\begin{aligned}
\mathrm{E} \sup _{0 \leqslant t \leqslant T}\left\|\int_{0}^{t} S(t-r) b(X(r, y)) \mathrm{d} W(r)\right\|^{p} & \leqslant C_{2} \int_{0}^{T}\left(1+\mathrm{E}\|X(r, y)\|^{p}\right) \mathrm{d} r \\
& \leqslant C_{3} T\left(1+\|y\|^{p}\right)
\end{aligned}
$$


for all $y \in H, T \in[0,1]$ and some constants $C_{2}, C_{3}$. As, obviously,

$$
\mathrm{E} \sup _{0 \leqslant t \leqslant T}\left\|\int_{0}^{t} S(t-r) f(X(r, y)) \mathrm{d} r\right\|^{p} \leqslant C_{4} T\left(1+\|y\|^{p}\right)
$$

we have obtained an estimate

$$
\mathrm{E}_{y} \sup _{0 \leqslant t \leqslant T}\left\|X_{t}-y\right\|^{p} \leqslant C_{5}\left(\sup _{0 \leqslant t \leqslant T}\|S(t) y-y\|^{p}+T\left(1+\|y\|^{p}\right)\right) .
$$

Take $\varepsilon>0$ and $x \in H$, let $V$ be an $\varepsilon^{1 / p}$-neighbourhood of $x$, then

$$
\|S(t) y-y\| \leqslant\left(\sup _{0 \leqslant t \leqslant 1}\|S(t)\|+1\right) \varepsilon^{1 / p}+\|S(t) x-x\|, \quad y \in V
$$

hence it is clear that we can find $T>0$ such that

$$
\sup _{y \in V} \mathrm{E} \sup _{0 \leqslant t \leqslant T}\left\|X_{t}-y\right\|^{p} \leqslant C_{6} \varepsilon
$$

and (A5) follows.

The next two examples are, strictly speaking, only particular cases of Example 3.1, but they seem to be worth being mentioned separately.

Example 3.2. Let us consider a stochastic semilinear heat equation

$$
\begin{gathered}
\frac{\partial}{\partial t} X(t, \xi)=\frac{\partial^{2}}{\partial \xi^{2}} X(t, \xi)-\alpha X(t, \xi)+F(X(t, \xi))+B(X(t, \xi)) \frac{\partial^{2} W}{\partial \xi \partial t} \\
t \geqslant 0, \quad \xi \in \mathbb{S}^{1}
\end{gathered}
$$

where $\mathbb{S}^{1}$ denotes the unit circle $\{z \in \mathbb{C} ;|z|=1\}, W_{t \xi}$ is a Brownian sheet on $\left[0, \infty\left[\times \mathbb{S}^{1}\right.\right.$, and $\alpha \in \mathbb{R}$. In a standard way one can transform the equation (3.3) into the form (3.1). Furthermore, the assumptions (A1)-(A4) are fulfilled in the space $H=L^{2}\left(\mathbb{S}^{1}\right)$ provided $F, B: \mathbb{R} \longrightarrow \mathbb{R}$ are Lipschitz, $B$ is bounded and there exists a constant $\lambda>0$ such that $|B(r)| \geqslant \lambda$ for any $r \in \mathbb{R}$ (cf. [33], Sect. 4). Using a rather complicated coupling technique $C$. Mueller showed recently that if there exists an invariant probability measure $\tilde{\mu}$ for (3.3) then the probability distributions $\mu_{t}$ of $X(t, \cdot)$ converge to $\tilde{\mu}$ in total variation (see [31], Theorem 1.2). The same result follows immediately from our Theorem 2.2. (There is a slight formal difference, as in [31] the solution to (3.3) is considered as a $\mathscr{C}\left(\mathbb{S}^{1}\right)$-valued Markov process, whilst in our approach as an $H$-valued process. But we know a priori that $\mu_{t}, \tilde{\mu}$ are supported by $\mathscr{C}\left(\mathbb{S}^{1}\right)$ and we have $\mathscr{C}\left(\mathbb{S}^{1}\right) \hookrightarrow H$ and $\mathscr{B}\left(\mathscr{C}\left(\mathbb{S}^{1}\right)\right) \subseteq \mathscr{B}(H)$, so using the Hahn decomposition it is easy to show that the two results are in fact identical.) 
Example 3.3. In this example we will consider a stochastic reaction-diffusion system written symbolically as

$$
\begin{gathered}
\frac{\partial u_{i}}{\partial t}=\frac{\partial^{2} u_{i}}{\partial x^{2}}+F_{i}\left(u_{1}, \ldots, u_{n}\right)+\eta_{i}, \\
u_{i}(t, 0)=u_{i}(t, 1)=0, \\
i=1, \ldots, n, \quad 0<x<1, \quad t \geqslant 0
\end{gathered}
$$

where $\eta_{i}$ stand for independent space-time white noises. Set $H=L^{2}\left((0,1) ; \mathbb{R}^{n}\right)$, let $W$ be a standard cylindrical Wiener process in $H$, define

$$
\operatorname{Dom}(A)=W^{2,2}\left((0,1) ; \mathbb{R}^{n}\right) \cap W_{0}^{1,2}\left((0,1) ; \mathbb{R}^{n}\right), \quad A u=\left(\begin{array}{ccc}
\frac{\partial^{2} u_{1}}{\partial x^{2}} & \ldots & 0 \\
\vdots & \ddots & \vdots \\
0 & \ldots & \frac{\partial^{2} u_{n}}{\partial x^{2}}
\end{array}\right)
$$

(where $W^{s, 2}$ denotes the usual Sobolev spaces), and

$$
f: H \longrightarrow H, \quad v \longmapsto\left(F_{1}(v(\cdot)), \ldots, F_{n}(v(\cdot))\right)
$$

We assume that the functions $F_{i}: \mathbb{R}^{n} \rightarrow \mathbb{R}, i=1, \ldots, n$, are Lipschitz continuous, so the mapping $f$ is Lipschitz as well. Hence the problem (3.4) can be treated as a stochastic evolution equation

$$
\mathrm{d} u=(A u+f(u)) \mathrm{d} t+\mathrm{dI}
$$

in $H$, which is a particular case of (3.1), therefore all the hypotheses (A1)-(A4) are satisfied. Moreover, suppose that the drift term is of a potential type, i.e. there exists a bounded from above function $U: \mathbb{R}^{n} \longrightarrow \mathbb{R}$ such that $\left(F_{1}, \ldots, F_{n}\right)=\nabla U$. Let us set

$$
\tilde{U}(x)=\int_{0}^{1} U(x(\xi)) \mathrm{d} \xi, \quad x \in H .
$$

Denote by $\nu=N\left(0,-\frac{1}{2} A^{-1}\right)$ the Gaussian measure on $H$ with zero mean and a (nuclear) covariance operator $-\frac{1}{2} A^{-1}$. By [43], Theorem 2 (cf. also [14], Theorem 4.1 , there exists a finite invariant measure for (3.5), namely

$$
\mathrm{d} \mu=\exp (\tilde{U}(\cdot)) \mathrm{d} \nu
$$

The behaviour of the Gaussian measure $\nu$ is well unclerstood, hence we know that if $C$ is an arbitrary nonempty open set in the space $\varphi_{0}\left([0,1] ; \mathbb{R}^{n}\right)$ of all $\mathbb{R}^{n}$-valued continuous functions $\varphi$ on $[0,1]$ with $\varphi(0)=\varphi(1)=0$ then $\mu(C)>0$ and thus 
by Theorem 2.2 the set $C$ is recurrent for the Markov process solving the equation (3.5). It seems to be difficult to obtain results on recurrence of sets in $\mathscr{C}_{0}\left([0,1] ; \mathbb{R}^{n}\right)$ by direct methods. In fact, up to our best knowledge, there is only T. Funaki's paper in which the case when $n=1$ or $2, F_{i} \equiv 0$ and Dirichlet boundary data in (3.4) are replaced by the Neumann ones was treated ([14], Theorem 5.1).

In previous examples we adopted the rather stringent assumption that the nonlinear term in the drift of the stochastic evolution equation is Lipschitz continuous. Now we turn to applications in which this assumption is relaxed.

Example 3.4. This example is based on a recent paper by A. ChojnowskaMichalik and B. Gołdys. We consider a Markov process defined by a stochastic differential equation

$$
\mathrm{d} X=(A X+g(X)) \mathrm{d} t+\mathrm{d} W
$$

in a separable Hilbert space $H$, supposing

(a) $A$ generates a $C_{0}$-semigroup $S(t)$ on $H$,

(b) $W$ is a (cylindrical) Wiener process in $H$ with a covariance operator $Q \in \mathscr{L}(H)$,

(c) $g: H \longrightarrow H$ is bounded and weakly continuous, that is

$$
\sup _{x \in H}\|g(x)\|<\infty, \quad\langle g(\cdot), y\rangle \in \mathscr{C}_{b}(H) \quad \text { for all } y \in H .
$$

Further we assume that

$$
\sup _{t \geqslant 0} \operatorname{Tr} Q_{t} \equiv \sup _{t \geqslant 0} \operatorname{Tr} \int_{0}^{t} S(r) Q S(r)^{*} \mathrm{~d} r<\infty,
$$

and

$$
\operatorname{Rng} S(t) \subseteq \operatorname{Rng} Q_{t}^{1 / 2}, \quad \operatorname{Ker} Q_{t}^{1 / 2}=\{0\}, \quad \int_{0}^{t}\left\|Q_{r}^{-1 / 2} S(r)\right\| \mathrm{d} r<\infty
$$

for any $t>0$. By the first inclusion in (3.8) the operators $S(t), t>0$, are HilbertSchmidt, but we will need more, namely

(d) There exists $r \in] 0,1[$ such that (3.2) holds for every $T>0$.

By [16], Theorem 2, the assumptions (a), (c), (d) yield that for any $y \in H$ there exists a martingale solution $X(\cdot, y)$ (in the sense of [7], Chapter 8) of (3.6) fulfilling $X(0, y)=y$, and the process $X(\cdot, y)$ has continuous sample paths. Further, this solution is unique in law (see [5], Theorem 4), therefore (3.6) defines a Markov process with continuous paths, and this process is strong Feller and irreducible ([5], Theorem 3 and Proposition 4, respectively). The assumption (A5) can be checked as in Example 3.1. 
Remark 3.1. In Example 3.4, we adopted the same hypotheses as used in the papers [16], [5]. It can be observed easily, however. that the following assumption: there exists $q \in] 0,1[$ such that

$$
\int_{0}^{t} r^{-q}\left\|S(r) Q^{1 / 2}\right\|_{\mathrm{HS}}^{2} \mathrm{~d} r<\infty, \quad t \geqslant 0
$$

may replace (d), and (3.7) may be relaxed to (3.9) with $q=0$. The assumption (3.7) in fact yields that there exists an invariant probability measure for (3.6) possessing a density $h$ with respect to the invariant measure $\nu$ of the Ornstein-Uhlenbeck process

$$
\mathrm{d} Z=A Z \mathrm{~d} t+\mathrm{d} W
$$

see [5], Theorem 5. Moreover, $h>0 \nu$-almost everywhere by [5], Proposition 5 . Consequently, the nonlinear terms $F_{i}$ in the equation (3.4) may be assumed to be only bounded continuous functions on $\mathbb{R}^{n}$, nevertheless, all the assertions on recurrence in Example 3.3 remain valid.

We conclude this section by discussing equations with unbounded drift coefficients (e.g. of polynomial growth), relying on results due to B. Maslowski.

Example 3.5. Let $\left(E,\|\cdot\|_{E}\right)$ be a separable Banach space continuously embedded into a separable Hilbert space $(H,\|\cdot\|)$, let $W$ be a (cylindrical) Wiener process in $H$ with a covariance operator $Q \in \mathscr{L}(H)$ such that $Q>0$. Let $A$ : $\operatorname{Dom}(A) \subseteq E \longrightarrow E$ be an infinitesimal generator of a strongly continuous semigroup $S(t)$ on $E$, let us assume that this semigroup can be extended to a $C_{0}$-semigroup $S_{0}(t)$ on $H$ (with a generator $A_{0}: \operatorname{Dom}\left(A_{0}\right) \longrightarrow H$ ). Further suppose that

$$
\int_{0}^{T}\left\|S_{0}(t) Q^{1 / 2}\right\|_{\mathrm{HS}}^{2} \mathrm{~d} s<\infty
$$

for any $T>0$. Under (3.10) the process

$$
Z(t)=\int_{0}^{t} S_{0}(t-r) \mathrm{d} W(r), \quad t \geqslant 0,
$$

in $H$ is well defined, we will assume that $Z$ has an $E$-valued modification with paths continuous in $E$. Let us consider a mapping $f: E \longrightarrow E$ locally Lipschitz continuous in the following sense: for any $N>0$ there exists $K_{N}<\infty$ such that

$$
\|f(x)-f(y)\| \leqslant K_{N}\|x-y\|, \quad\|f(x)-f(y)\|_{E} \leqslant K_{N}\|x-y\|_{E}
$$


whenever $x, y \in E$ satisfy $\|x\|_{E},\|y\|_{E} \leqslant N$. Let there exist a nondecreasing function $a: \mathbb{R}_{+} \longrightarrow \mathbb{R}_{+}$such that for any $x \in \operatorname{Dom}(A)$ one can find $x^{*} \in \partial\|x\|_{E}$ with the property

$$
\left\langle A x+f(x+y), x^{*}\right\rangle_{E, E^{*}} \leqslant a\left(\|y\|_{E}\right), \quad y \in E,
$$

where $\partial\|x\|_{E}$ denotes the subdifferential of the norm $\|\cdot\|_{E}$ at the point $x$. By [7], Theorem 7.10, there exists a unique mild solution $X(\cdot, y)$ of the equation

$$
\mathrm{d} X=(A X+f(X)) \mathrm{d} t+\mathrm{d} W
$$

such that $X(0, y)=y, y \in E$ arbitrary, thus (3.12) defines a Markov process $X$ in $E$, moreover this process fulfils (A1). Assume additionally

$$
\lim _{T \rightarrow 0+} \mathrm{E} \sup _{0 \leqslant t \leqslant T}\|Z(t)\|_{E}=0
$$

and

$$
\lim _{T \rightarrow 0+} \sup _{x \in K^{*}} \int_{0}^{T} \mathrm{E} a\left(\|S(s) x+Z(s)\|_{E}\right) \mathrm{d} s=0
$$

for any compact set $K$ in $E$, then (A4) is satisfied. Indeed, by the definition of a mild solution we have

$$
X(t, y)=S(t) y+\int_{0}^{t} S(t-s) f(X(s, y)) \mathrm{d} s+Z(t),
$$

so setting $v^{y}(t)=X(t, y)-S(t) y-Z(t)$ we have $v^{y}(0)=0$ and

$$
v^{y}(t)=\int_{0}^{t} S(t-s) f\left(v^{y}(s)+S(s) y+Z(s)\right) \mathrm{d} s .
$$

Using (3.11) and proceeding as in the proof of the quoted theorem from [7] we arrive at an estimate

$$
\left\|v^{y}(t)\right\|_{E} \leqslant \int_{0}^{t} a\left(\|S(s) y+Z(s)\|_{E}\right) \mathrm{d} s,
$$

therefore

$$
\begin{aligned}
\sup _{y \in K} \mathrm{E} \sup _{0 \leqslant t \leqslant T}\|X(t, y)-y\|_{E} \leqslant & \sup _{0 \leqslant t \leqslant T} \sup _{y \in K}\|S(t) y-y\|_{E}+\mathrm{E} \sup _{0 \leqslant t \leqslant T}\|Z(t)\|_{E} \\
& +\sup _{y \in K} \int_{0}^{T} \mathrm{E} a\left(\|S(t) y+Z(t)\|_{E}\right) \mathrm{d} t
\end{aligned}
$$


and by (3.13), (3.14) the assumption (A4) follows. Furthermore, assume that

$$
S_{0}(t) \in \mathscr{L}(H, E), \quad \int_{0}^{t}\left\|S_{0}(r)\right\|_{\mathscr{L}(H, E)}^{2} \mathrm{~d} r<\infty
$$

for any $t>0$, then the process $X$ is irreducible in $E$ by [26], Proposition 2.11 . For $X$ to be strong Feller a bit technical assumption is needed. Let there exist an orthonormal basis $\left\{e_{n}\right\}$ of $H,\left\{e_{n} ; n \geqslant 1\right\} \subseteq \operatorname{Dom}\left(A_{0}\right)$, with the following properties: Denote by $P_{n}$ the orthogonal projection on the linear span of $\left\{e_{1}, \ldots, e_{n}\right\}$, and set

$$
\begin{gathered}
A_{n}=P_{n} A_{0} P_{n}, \quad S_{n}(t)=\mathrm{r}^{A, n}, \\
Q_{t}=\int_{0}^{t} S_{0}(r) Q S_{0}(r)^{*} \mathrm{~d} r, \quad Q_{t, n}=\int_{0}^{t} S_{n}(r) P_{n} Q P_{n} S_{n}(r)^{*} \mathrm{~d} r .
\end{gathered}
$$

Suppose that

$$
\operatorname{Rng} S_{0}(t) \subseteq \operatorname{Rng} Q_{t}^{1 / 2}
$$

for any $t>0$, and set further

$$
B_{t}=Q_{t}^{-1 / 2} S_{0}(t), \quad B_{t, n}=Q_{t, n}^{-1 / 2} S_{n}(t) P_{n} .
$$

We assume that for any $T>0$ one can find constants $C<\infty$ and $\alpha \in] 0,1[$ such that

$$
\begin{aligned}
& \sup _{n \geqslant 1}\left\|S_{n}(t)\right\|_{\mathscr{L}(H)}+\left\|S_{0}(t)\right\|_{\mathscr{L}(H)} \leqslant C, \quad 0 \leqslant t \leqslant T, \\
& \sup _{n \geqslant 1}\left\|B_{t, n}\right\|_{\mathscr{L}(H)}+\left\|B_{t}\right\|_{\mathscr{L}(H)} \leqslant \frac{C}{t^{\alpha}}, \quad 0 \leqslant t \leqslant T,
\end{aligned}
$$

and

$$
\lim _{n \rightarrow \infty} \sup _{t \in[0, T]}\left\|S_{n}(t) P_{n} x-S_{0}(t) x\right\|=0, \quad x \in \operatorname{Dom}\left(A_{0}\right) .
$$

Then $X$ is a strong Feller process in $E$ ([26], Proposition 2.3), hence for the Markov process $X$ in the space $E$ the assumptions (A1)-(A4) are satisfied.

The machinery developed above may look rather cumbersome, but it covers the popular example of a stochastic heat equation with a polynomial drift. 
Example 3.6. We consider the equation

$$
\begin{aligned}
\frac{\partial u}{\partial t}(t, x) & =\frac{\partial^{2} u}{\partial x^{2}}(t, x)+p(u(t, x))+\dot{W}, \\
u(t, 0) & =u(t, \pi)=0, \quad t \geqslant 0, \quad 0<x<\pi,
\end{aligned}
$$

where

$$
p(r)=\sum_{n=0}^{2 k+1} b_{n} r^{n}, \quad b_{2 k+1}<0, \quad r \in \mathbb{R},
$$

is a polynomial of odd degree with a negative leading coefficient; the Wiener process $W$ will be specified later. We intend to use the results recalled in the previous example. To this end, set $H=L^{2}((0, \pi)), E=\mathscr{C}_{0}([0, \pi])$, and

$$
\operatorname{Dom}\left(A_{0}\right)=W^{2,2}((0, \pi)) \cap W_{0}^{1,2}((0, \pi)), \quad A_{0} v=\frac{\partial^{2} v}{\partial x^{2}},
$$

let $A$ be the part of $A_{0}$ in $E$. Then $A, A_{0}$ give rise to contractive semigroups in $E$, $H$, respectively, so in particular we have $\left\langle A x, x^{*}\right\rangle \leqslant 0$ for any $x \in E, x^{*} \in \partial\|x\|_{E}$; moreover, (3.15) is valid. Denote by $\left\{e_{n}\right\}$ the orthonormal basis of $H$ consisting of the eigenvectors of $A_{0}$, i.e. let

$$
A_{0}=-\sum_{n=1}^{\infty} n^{2} e_{n} \otimes e_{n}
$$

Let $W$ be a Wiener process in $H$ with a covariance operator $Q$ of the particular form

$$
Q=\sum_{n=1}^{\infty} \lambda_{n} e_{n} \otimes e_{n}
$$

where $0<\ldots \lambda_{n} \leqslant \lambda_{1}<\infty$ (that is, $Q>0$ ). Then (3.10) holds and the process $Z$ is defined. In [26] (Examples 3.5 and 3.7) it is shown that (3.16)-(3.19) are fulfilled provided

$$
\sup _{i \geqslant 1} \sqrt{\frac{i^{2}}{\lambda_{i}\left\{\exp \left(2 i^{2} t\right)-1\right\}}} \leqslant \frac{c}{t^{\alpha}}, \quad 0 \leqslant t \leqslant T,
$$

for a constant $c$. Define a mapping $f: E \longrightarrow E$ by $f(v)=p(v(\cdot)), v \in E$, then $f$ is locally Lipschitz in the required sense, moreover (3.11) is fulfilled with a function $a(r)=K\left(r^{2 k+1}+1\right), r \in \mathbb{R}_{+}$, where $\left.K \in\right] 0, \infty[$ is an appropriate constant. 
It remains to show that $Z$ has a modification with paths continuous in $E$ and fulfils (3.13), (3.14). First, assume in addition that

$$
\operatorname{Tr} Q=\sum_{n=1}^{\infty} \lambda_{n}<\infty
$$

(It is worth noticing that assumptions (3.22) and (3.21) are compatible, e.g. we can take $\lambda_{i}=i^{-(1+\varepsilon)}, 0<\varepsilon<1$.) Taking into account that the spaces $\operatorname{Dom}\left(\left(-A_{0}\right)^{\delta}\right)$ endowed with the graph norm are continuously emberded into $E$ if $\delta>\frac{1}{4}$ we see that Theorem 1.1 from [35] yields that the process $Z$ is continuous in $E$ and satisfies the estimate

$$
\mathrm{E} \sup _{0 \leqslant t \leqslant T}\|Z(t)\|_{E}^{q} \leqslant C_{q} T
$$

for any $q>4$. Next, let us consider the more interesting case $\lambda_{i}=1$, that is $Q=I$. Then (3.21) holds, hence the assumptions (A2), (A3) are fulfilled, and it is known that (A1) holds as well (see e.g. [7], Example 5.21). By [32], Corollary 2.5, we have

$$
\mathrm{E} \sup _{0 \leqslant 1 \leqslant T}\|Z(t)\|_{E}^{q} \leqslant C_{q, \varepsilon} T^{\frac{q}{4}-2-\varepsilon}
$$

for any $q>8$ and $\varepsilon \in] 0, \frac{1}{4} q-2[$. Due to the particular form of the function $a$ we obtain at once that either of the estimates (3.23), (3.24) implies (3.14), therefore the Markov process in $E$ defined by the equation (3.20) fulfils all the assumptions (A1)-(A4).

\section{Proof of Theorem 2.1}

First, we establish an auxiliary lemma.

Lemma 4.1. Let the assumptions (A1), (A2) and (A4) be fulfilled. Let $F \subseteq H$ be a closed set, let $\tau$ be the first hitting time of $F$. that is,

$$
\tau(\omega)=\inf \left\{t>0 ; X_{t}(\omega) \in F\right\} .
$$

Let $g \in \mathbf{B}(H)$ and $t>0$ be arbitrary. Then the function

$$
x \longmapsto \mathrm{E}_{x}\left[X_{\{\tau>t\}} g\left(X_{t}\right)\right]
$$

is continuous on $H \backslash F$. 
Remark 4.1. i) An analogous proof yields that the function $x \longmapsto \mathrm{P}_{x}\{\tau=\infty\}$ is continuous on $H \backslash F$.

ii) One cannot use directly Theorem 13.1 from [11] as its proof uses implicitly the local compactness of the state space, nevertheless, the idea of the proof remains the same.

Proof. Fix $t>0, g \in \mathbf{B}(H)$ and set

$$
J(x)=\mathrm{E}_{x}\left[\chi_{\{\tau>t\}} g\left(X_{t}\right)\right], \quad x \in H .
$$

For any $x \in H$ and $s \in] 0, t[$ we have

$$
\begin{gathered}
J(x)=\mathrm{E}_{x}\left[\chi_{\{\tau>t\}} g\left(X_{t}\right)\right]=\mathrm{E}_{x}\left[\chi_{\{\tau>s\}} \theta_{s}\left(\chi_{\{\tau>t-s\}} g\left(X_{t-s}\right)\right)\right]= \\
=\mathrm{E}_{x}\left[\theta_{s}\left(\chi_{\{\tau>t-s\}} g\left(X_{t-s}\right)\right)\right]-\mathrm{E}_{x}\left[\chi_{\{\tau \leqslant s\}} \theta_{s}\left(\chi_{\{\tau>t-s\}} g\left(X_{t-s}\right)\right)\right] \equiv \\
\equiv J_{1}(s, x)-J_{2}(s, x) .
\end{gathered}
$$

Assume that there exists $w \in H \backslash F$ such that $J$ is not continuous at the point $w$, that is, there exist $\varepsilon>0, w_{n} \in H \backslash F, w_{n} \longrightarrow w$, satisfying

$$
\left|J\left(w_{n}\right)-J(w)\right|>\varepsilon, \quad n \geqslant 1 .
$$

Denote by $C$ the compact set $\left\{w_{n} ; n \in \mathbb{N}\right\} \cup\{w\}$, then $\varrho \equiv \operatorname{dist}(C, F)>0$. First, let us realize that

$$
\begin{aligned}
\left|J_{2}\left(s, w_{n}\right)-J_{2}(s, w)\right| & \leqslant\left|J_{2}\left(s, w_{n}\right)\right|+\left|J_{2}(s, w)\right| \\
& \leqslant\|g\|_{\infty}\left\{\mathrm{P}_{w_{n}}\{\tau \leqslant s\}+\mathrm{P}_{w}\{\tau \leqslant s\}\right\} \\
& \leqslant 2\|g\|_{\infty} \sup _{y \in C} \mathrm{P}_{y}\{\tau \leqslant s\} .
\end{aligned}
$$

Using the assumption (A4) we can find $u>0$ such that

$$
\sup _{y \in C} P_{y}\left\{\sup _{0 \leqslant v \leqslant u}\left\|X_{v}-y\right\|>\frac{\varrho}{2}\right\} \leqslant \frac{\varepsilon}{4\|g\|_{\infty}} .
$$

Obviously, this estimate means that

$$
\sup _{y \in C} \mathrm{P}_{y}\{\tau \leqslant u\} \leqslant \frac{\varepsilon}{4\|g\|_{\infty}} .
$$

Further, set

$$
G_{s}(y)=\mathrm{E}_{y} \chi_{\{\tau>t-s\}} g\left(X_{t-s}\right), \quad y \in H .
$$


Obviously, $G_{s}$ is a bounded Borel function on $H$, according to the Markov property (see the formula (5.42) of [10]) we obtain

$$
J_{1}(s, x)=\mathrm{E}_{x}\left[\mathrm{E}_{X(s)} \chi_{\{\tau>t-s\}} g\left(X_{t-s}\right)\right]=\mathrm{E}_{x} G_{s}\left(X_{s}\right)=\int_{H} G_{s}(z) P(s, x, \mathrm{~d} z),
$$

hence $J_{1}(s, \cdot) \in \mathscr{C}_{b}(H)$ for all $\left.s \in\right] 0, t[$ by the strong Feller property. Thus

$$
\left|J_{1}\left(u, w_{n}\right)-J_{1}(u, w)\right| \leqslant \frac{\varepsilon}{2}
$$

for all $n$ sufficiently large, which together with (4.2) yields a contradiction with the inequality (4.1). Lemma 4.1 follows.

Pro of of The or em 2.1. Let $K$ be a nonrecurrent compact set in $H$. First we will prove, modifying slightly the proof of Lemma 3.1 from [20], that

$$
\sup _{x \in H} \int_{0}^{\infty} P(t, x, K) \mathrm{d} t<\infty .
$$

Let $\tau$ be the first hitting time of $K$. By assumption, there exists $x_{0} \notin K$ such that

$$
\mathrm{P}_{x_{0}}\{\tau=\infty\}=\mathrm{P}_{x_{0}}\left\{\omega ; X_{t}(\omega) \notin I \forall t \geqslant 0\right\}>0 .
$$

By Lemma 4.1, P. $\{\tau=\infty\}$ is continuous on $H \backslash K$, hence we can find an open set $V \ni x_{0}$ such that

$$
\inf _{x \in V} \mathrm{P}_{x}\{\tau=\infty\} \equiv \alpha>0 .
$$

Let us fix $T>0$ arbitrary. Let $y \in K$; applying the Markov property (cf. formula (5.41) from [10]) we obtain

$$
\begin{aligned}
\mathrm{P}_{y}\{\omega ; & \left.\int_{0}^{\infty} \chi_{K}\left(X_{t}(\omega)\right) \mathrm{d} t<T\right\} \\
& \geqslant \mathrm{P}_{y}\left(\left\{\omega ; X_{T} \in V\right\} \cap\left\{\omega ; X_{t}(\omega) \notin K \forall t \geqslant T\right\}\right) \\
& =\mathrm{P}_{y}\left(\left\{X_{T} \in V\right\} \cap \theta_{T}\left\{X_{t} \notin K \forall t \geqslant 0\right\}\right) \\
& =\int_{\{X(T) \in V\}} \mathrm{P}_{X(T)}\{\tau=\infty\} \mathrm{dP}_{y} \geqslant \alpha \int_{\{X(T) \in V\}} \mathrm{dP}_{y} \\
& =\alpha P(T, y, V) \geqslant \alpha \inf _{z \in K} P(T, z, V) \equiv q,
\end{aligned}
$$

where we have used the fact that $\mathrm{P}_{X(T)}\{\tau=\infty\} \geqslant a$ on the set $\left\{X_{T} \in V\right\}$ by (4.4). Due to (A3), (A2) and the compactness of $K$ we have $q>0$. Hence

$$
\sup _{y \in K} \mathrm{P}_{y}\left\{\omega ; \int_{0}^{\infty} \chi_{K}\left(X_{t}(\omega)\right) \mathrm{d} t \geqslant T\right\} \leqslant 1-q<1 .
$$


Let us set

$$
\varkappa=\inf \left\{t \geqslant 0 ; \int_{0}^{t} \chi_{K}\left(X_{s}\right) \mathrm{d} s=T\right\}
$$

note that $\varkappa$ is a stopping time with respect to $\left(\mathscr{F}_{t}\right)$. As $K$ is closed and (A1) is assumed we obtain $X(\varkappa) \in K$ on the set $\{\varkappa<\infty\}$. Let $y \in \Lambda$, taking into account (4.5) and the inclusions

$$
\left\{\int_{0}^{\infty} \chi_{K}\left(X_{t}\right) \mathrm{d} t>T\right\} \subseteq\{\varkappa<\infty\} \subseteq\left\{\int_{0}^{\infty} \chi_{K}\left(X_{t}\right) \mathrm{d} t \geqslant T\right\}
$$

we obtain

$$
\begin{aligned}
& \mathrm{P}_{y}\left\{\omega ; \int_{0}^{\infty} \chi_{K}\left(X_{t}\right) \mathrm{d} t>2 T\right\} \\
& \quad=\mathrm{P}_{y}\left(\{\varkappa<\infty\} \cap\left\{\int_{\varkappa}^{\infty} \chi_{K}\left(X_{t}\right) \mathrm{d} t>T\right\}\right) \\
& \quad=\mathrm{P}_{y}\left(\{\varkappa<\infty\} \cap \theta_{\varkappa}\left\{\int_{0}^{\infty} \chi_{K}\left(X_{t}\right) \mathrm{d} t>T\right\}\right) \\
&=\int_{\{\varkappa<\infty\}} \mathrm{P}_{X(\varkappa)}\left\{\int_{0}^{\infty} \chi_{K}\left(X_{t}\right) \mathrm{d} t>T\right\} \mathrm{dP} y \\
& \leqslant(1-q)^{2}
\end{aligned}
$$

by the strong Markov property. Thus, by induction,

$$
\mathrm{P}_{y}\left\{\int_{0}^{\infty} \chi_{\kappa}\left(X_{t}\right) \mathrm{d} t>k T\right\} \leqslant(1-q)^{k}, \quad k \in \mathbb{N}
$$

for all $y \in K$. It follows easily that (4.6) holds for every $y \in H$; note that $q$ does not depend on $y$.

Finally, take $x \in H$ arbitrary, then

$$
\begin{aligned}
\int_{0}^{\infty} P(t, x, K) \mathrm{d} t & =\mathrm{E}_{x} \int_{0}^{\infty} \chi_{K}\left(X_{t}\right) \mathrm{d} t \\
& \leqslant \sum_{k=1}^{\infty}(k T) \mathrm{P}_{x}\left\{(k-1) t<\int_{0}^{\infty} \chi_{K}\left(X_{t}\right) \mathrm{d} t \leqslant k T\right\} \\
& \leqslant \sum_{k=1}^{\infty}(k T) \mathrm{P}_{x}\left\{\int_{0}^{\infty} \chi_{K}\left(X_{t}\right) \mathrm{d} t>(k-1) T\right\} \\
& \leqslant T \sum_{k=1}^{\infty} k(1-q)^{k-1}<\infty .
\end{aligned}
$$


Further, let $\mu$ be a finite invariant measure. The proof that $\mu(K)=0$ is standard, as we have

$$
\lim _{T \rightarrow \infty} \frac{1}{T} \int_{0}^{T} P(t, x, K) \mathrm{d} t=0
$$

for all $x \in H$ by (4.3), and due to invariantness of $\mu$ we obtain

$$
\begin{aligned}
\mu(K) & =\frac{1}{T} \int_{0}^{T} \mu(K) \mathrm{d} t=\frac{1}{T} \int_{0}^{T} \int_{H} P(t, y, K) \mathrm{d} \mu(y) \mathrm{d} t \\
& =\int_{H}\left(\frac{1}{T} \int_{0}^{T} P(t, y, K) \mathrm{d} t\right) \mathrm{d} \mu(y) \stackrel{T \rightarrow \infty}{\longrightarrow} 0 ;
\end{aligned}
$$

note that the use of the dominated convergence theorem is justified by the finiteness of $\mu$.

Now, assume that $\varphi(K)=P(1,0, K)>0$. Let $L \subseteq H$ be an arbitrary compact, we want to prove that

$$
\mathrm{P}_{x}\left\{\sup \left\{t \geqslant 0 ; X_{t} \in L\right\}<\infty\right\}=1
$$

for all $x \in H$. Set

$$
h(x)=\int_{0}^{\infty} P(s, x, K) \mathrm{d} s, \quad x \in H,
$$

then $h \in \mathbf{B}(H)$ by (4.3), moreover, we have $h>0$ on $H$ due to the equivalence of transition probabilities. Hence (4.7) follows by [17], Proposition 2.2, for completeness we repeat the simple proof: The function $h$ is obviously lower semicontinuous, thus the sets $H_{n}=\left\{y \in H ; h(y)>\frac{1}{n}\right\}$ are open and $H_{n} \uparrow H$. As $L$ is compact we can find $m \in \mathbb{N}$ such that $L \subseteq H_{m}$. Fix $x \in H$, denote by $\sigma$ the first hitting time of $L$, and let $\sigma(k), k \in \mathbb{N}$, stand for the first hitting time of $L$ after $k$, i.e. $\sigma(k)=k+\theta_{k} \sigma$. Taking into account that $X(\sigma(k)) \in L \subseteq H_{m} \mathrm{P}_{x}$-almost surely on the set $\{\sigma(k)<\infty\}$ we arrive at

$$
\begin{aligned}
\frac{1}{m} \mathrm{P}_{x}\{\omega ; \sigma(k)<\infty\} & \leqslant \mathrm{E}_{x} \chi_{\{\sigma(k)<\infty\}} h(X(\sigma(k))) \\
& =\mathrm{E}_{x} \int_{0}^{\infty} \chi_{\{\sigma(k)<\infty\}} P(s, X(\sigma(k)), K) \mathrm{d} s \\
& =\mathrm{E}_{x} \int_{0}^{\infty} \chi_{\{\sigma(k)<\infty\}} \mathrm{E}_{x}\left(\chi_{K}(X(\sigma(k)+s)) \mid \mathscr{F}_{\sigma(k)}\right) \mathrm{d} s \\
& =\mathrm{E}_{x} \chi_{\{\sigma(k)<\infty\}} \int_{\sigma(k)}^{\infty} \mathrm{E}_{x}\left(\chi_{K}(X(v)) \mid \mathscr{F}_{\sigma(k)}\right) \mathrm{d} v \\
& \leqslant \mathrm{E}_{x} \int_{k}^{\infty} \chi_{K}(X(v)) \mathrm{d} v=\int_{k}^{\infty} P(v, x, K) \mathrm{d} v \stackrel{k \rightarrow \infty}{\longrightarrow} 0 .
\end{aligned}
$$


As the sequence of sets $(\{\sigma(k)<\infty\}, k \in \mathbb{N})$ is decreasing we obtain that for $\mathrm{P}_{x^{-}}$ almost any $\omega \in \Omega$ there exists $k$ such that $\sigma(k)(\omega)=\infty$, which completes the proof of (4.7). Now

$$
\lim _{t \rightarrow \infty} P(t, x, L)=0
$$

follows easily.

Remark 4.2. The proof of Theorem 2.1 remains valid without the assumption (A3) provided all measures $P(t, x, \cdot), t>0, x \in H$, are equivalent. We have only to show how to find an open set $V \subseteq H$ satisfying

$$
\varphi(V)>0 \quad \text { and } \quad \inf _{x \in V} \mathrm{P}_{x}\{\tau=\infty\}>0 .
$$

By the equivalence of transition probabilities there exists a closed set $S \subseteq H$ such that $P(t, x, S)=1, t>0, x \in H$, and $\varphi(U)>0$ for any open set $U$ fulfilling $U \cap S \neq \emptyset$. We suppose that $\mathrm{P}_{x_{1}}\{\tau=\infty\}>0$, thus also

$$
\begin{aligned}
0 & <\mathrm{P}_{x_{0}}\left(\theta_{1}\{\tau=\infty\}\right)=\mathrm{P}_{x_{0}} \mathrm{P}_{X(1)}\{\tau=\infty\}=\int_{H} \mathrm{P}_{y}\{\tau=\infty\} P\left(1, x_{0}, \mathrm{~d} y\right) \\
& =\int_{S} \mathrm{P}_{y}\{\tau=\infty\} P\left(1, x_{0}, \mathrm{~d} y\right) .
\end{aligned}
$$

Consequently, there exists $y_{0} \in S$ such that $\mathrm{P}_{y_{0}}\{\tau=\infty\}>0$, obviously we can take $y_{0} \notin K$, and (4.8) follows.

\section{Proof of Theorem 2.2}

In the sequel, we will often assume the equivalence of transition probabilities, that is

(E) All probability measures $P(t, x, \cdot), t>0, x \in H$, are equivalent.

As we have already mentioned, (E) is an easy consequence of (A2) and (A3). The following interesting result is basic for this section.

Lemma 5.1. Let the assumptions (A1), (A2) and (E) be satisfied. Let the filtration $\left(\mathscr{F}_{t}\right)$ fulfil the usual conditions. Assume that there exists a recurrent compact set $K$ in $H$. Then all Borel sets $B \in \mathscr{B}(H)$ such that $\varphi(B)>0$ are recurrent.

Remark 5.1. We need the usual conditions for the filtration $\left(\mathscr{F}_{t}\right)$ to be fulfilled only to ensure that the first hitting time of $B$ is a stopping time. If the additional assumption upon the filtration $\left(\mathscr{F}_{t}\right)$ is omitted then the proof yields that all closed sets $F$ with $\varphi(F)>0$ are recurrent; only this form of Lemma 5.1 will be used in the sequel. 
Remark 5.2. S. R. Meyn and R. L. Tweedie proved recently ([29], Theorem 1.1) that if the state space is locally compact separable metric then for a Markov process $\Phi$ subject to some mild restrictions the following are equivalent:

(i) $\Phi$ is Harris recurrent (hence there exists a $\sigma$-finite invariant measure for $\Phi$ ).

(ii) there exists a $\sigma$-finite measure $q$ such that all Borel sets $A$ with $q(A)>0$ are recurrent for $\Phi$.

Their proof seems to be virtually independent of the topological assumptions on the state space. Nevertheless, in our particular situation we prove the same result using a different argument.

Proof. Fix an arbitrary $B \in \mathscr{B}(H)$ with $\varphi(B)>0$. Denote by

$$
\mathfrak{m}=\inf \left\{t>0 ; X_{t} \in B\right\}
$$

the first hitting time of $B$. We want to prove that

$$
\mathrm{P}_{x}\{\mathfrak{m}<\infty\}=1
$$

for any $x \in H$. By the equivalence of transition probabilities $P\left(\frac{1}{2}, y, B\right)>0$ for all $y \in H$, hence by (A2) and the compactness of $K$ we obtain

$$
\inf _{x \in K} P_{x}\{m \leqslant 1\} \geqslant \inf _{x \in K} P\left(\frac{1}{2}, x, B\right) \equiv q>0 .
$$

Let $\tau$ be the first hitting time of $F$ and define by induction

$$
\begin{aligned}
\tau_{1} & =\tau . \\
\tau_{n+1} & =\mathrm{uf}\left\{t>\tau_{i 2}+2, X_{t} \in I\right\}=\tau_{n}+2+\theta_{\tau_{n}+2} \tau .
\end{aligned}
$$

By the assumption of the lemua $\tau_{n}<\infty$ and $X\left(\tau_{i j}\right) \in K P_{i x}$-almost surely, $x \in H$, for any $n \in \mathbb{N}$. Set

$$
A_{n}=\left\{\omega \in \Omega ; \tau_{n} \leqslant \mathrm{~m} \leqslant \tau_{n}+1\right\}=\theta_{\tau_{1}}\{\mathrm{~m} \leqslant 1\},
$$

then $A_{n} \in \mathscr{F}_{\tau_{n}+1} \subseteq \mathscr{F}_{\tau_{n} ;}$ anc: we have

$$
\begin{aligned}
& \sum_{n=1}^{\infty} \mathrm{P}_{x}\left(A_{n} \mid \mathscr{\mathcal { F }}_{\tau_{n}}\right)=\sum_{n=1}^{\infty} \mathrm{P}_{x}\left(\theta_{\tau_{n}}\{\mathrm{~m} \leqslant 1\} \mid \mathscr{\mathscr { K }}_{\tau_{n}}\right) \\
& =\sum_{i=1}^{\infty} P_{X\left(\tau_{n}\right)}\{m \leqslant 1\} \geqslant \sum_{n=1}^{\infty} \inf _{y \in K} P_{y}\{\mathfrak{m} \leqslant 1\} \\
& \geqslant \sum_{n=1}^{\infty} q=+\infty \quad P_{x} \text {-alnost surely, }
\end{aligned}
$$


$x \in H$ arbitrary. Therefore by the generalized Borel-Cantelli lemma (see e.g. [36], Corollary VII.5.2) $\mathrm{P}_{x}$-almost any $\omega \in \Omega$ lies in some $A_{n}$, so $\mathfrak{m}(\omega) \leqslant \tau_{n}(\omega)+1<\infty$.

Lemma 5.2. Let $\Gamma: H \times \mathscr{B}(H) \longrightarrow[0,1]$ be a strong Feller Markov kernel such that all the measures $\Gamma(y, \cdot), y \in H$, are equivalent. Assume that $\gamma \neq 0$ is a subinvariant measure for $\Gamma$, i.e.

$$
\Gamma^{*} \gamma(A) \equiv \int_{H} \Gamma(x, A) \mathrm{d} \gamma(x) \leqslant \gamma(A)
$$

for all $A \in \mathscr{B}(H)$. Let there exist a set $R \in \mathscr{B}(H)$ satisfying

$$
0<\Gamma^{*} \gamma(R) \leqslant \gamma(R)<\infty
$$

Then $\gamma$ is a $\sigma$-finite Radon measure.

Note that if $\gamma$ is a priori known to be $\sigma$-finite then $R$ fulfilling (5.1) always exists, as $\Gamma^{*} \gamma(H)=\gamma(H)>0$. Further, recall that $\Gamma$ is strong Feller if $\Gamma(\cdot, A) \in \mathscr{C}_{b}(H)$ for any $A \in \mathscr{B}(H)$.

Proof. First we prove that $\gamma$ is locally finite. Take $x \in H$ arbitrary, by (5.1) we have

$$
\int_{H} \Gamma(y, R) \mathrm{d} \gamma(y)>0
$$

hence there exists $y_{0} \in H$ such that $\Gamma\left(y_{0}, R\right)>0$. Taking into account that the measures $\Gamma\left(y_{0}, \cdot\right)$ and $\Gamma(x, \cdot)$ are equivalent we see that $\delta \equiv \Gamma(x, R)>0$, so by the strong Feller property we can find an open neighbourhood $V \ni x$ such that $\Gamma(y, R) \geqslant \delta / 2$ for all $y \in V$. Thus

$$
\infty>\int_{H} \Gamma(y, R) \mathrm{d} \gamma(y) \geqslant \int_{V} \Gamma(y, R) \mathrm{d} \gamma(y) \geqslant \frac{\delta}{2} \gamma(V) .
$$

So we have established that $\gamma$ is locally finite; as $H$ is separable (hence Lindelöf) $\gamma$ is also $\sigma$-finite. By the well-known Ulam theorem the restriction of $\gamma$ to any open set of finite measure is Radon, hence $\gamma$ is Radon by Corollary 12.4 from [15].

We are prepared to present the first construction of a $\sigma$-finite invariant measure, following the procedure proposed in [38] (see also [3]). Define by

$$
U(x, \cdot)=\int_{0}^{\infty} \mathrm{e}^{-t} P(t, x, \cdot) \mathrm{d} t, \quad x \in H
$$


the resolvent kernel. If (E) is satisfied then the process $X$ is $\varphi$-irreducible, that is

$$
\sum_{n=1}^{\infty} U^{n}(x, A)=\int_{0}^{\infty} P(t, x, A) \mathrm{d} t>0
$$

holds for all $x \in H$ and $A \in \mathscr{B}(H)$ satisfying $\varphi(A)=P(1,0, A)>0$. Now, Theorem 4 from [13] states that $\varphi$-irreducibility yields the existence of a $\sigma$-finite measure $\mu \neq 0$ subinvariant with respect to $U$, that is, $U^{*} \mu \leqslant \mu$, where

$$
U^{*} \mu(\cdot)=\int_{H} U(y, \cdot) \mathrm{d} \mu(y) .
$$

Lemma 2 in [38] says that if there exists a set $Q \in \mathscr{B}(H)$ such that $\mu(Q)<\infty$ and

$$
\inf _{x \in H} \int_{0}^{\infty} P(t, x, Q) \mathrm{d} t=+\infty
$$

then $U^{*} \mu=\mu$. (Indeed, $\mu-U^{*} \mu$ is a well-defined nomnegative measure and

$$
\begin{aligned}
\int_{H} \sum_{n=1}^{k} U^{n}(x, Q) \mathrm{d}\left(\mu-U^{*} \mu\right)(x) & =U^{*} \mu(Q)-\left(U^{k+1}\right)^{*} \mu(Q) \\
& \leqslant U^{*} \mu(Q) \leqslant \mu(Q)<\infty
\end{aligned}
$$

hence passing $k \rightarrow \infty$ we obtain $\mu-U^{*} \mu=0$ by (5.2).) Therefore by Lemma 1 of [2] we obtain $P_{t}^{*} \mu=\mu, t>0$, thus $\mu$ is an invariant measure for $X$. (Only processes with values in locally compact spaces are considered in [2], but the proof of Lemma 1 uses only the fact that the $\sigma$-algebra $\mathscr{B}(H)$ is countably generated.) So we have

Lemma 5.3. Let (A1), (E) and (5.2) be fulfilled. Then there exists a $\sigma$-finite invariant measure $\mu$ for the process $X$. Up to a multiplicative constant, $\mu$ is the unique $\sigma$-finite invariant measure.

Proof. The existence of a $\sigma$-finite invariant measure was established above. uniqueness follows e.g. from Proposition A.1 in [28].

In what follows, $\mu$ will always denote the $\sigma$-finite measure provided by the above construction. We will need, in fact, a stronger form of (5.2), namely

(C) There exists a set $V \in \mathscr{B}(H)$ such that $\mu(V)<x$, and

$$
\mathrm{P}_{x}\left\{\omega \in \Omega ; \int_{0}^{\infty} \chi_{V}\left(X_{t}(\omega)\right) \mathrm{d} t=+\infty\right\}=1
$$

holds for any $x \in H$. 
It remains to show that the assumptions of Theorem 2.2 imply (C); this will be a consequence of the following proposition (inspired by Theorem 1 in [1]).

Proposition 5.4. Let the assumptions (A1)-(A4) be fulfilled. Assume that there exists a recurrent compact set $K$ in $H$. Then

$$
\mathrm{P}_{x}\left\{\omega \in \Omega ; \int_{0}^{\infty} \chi_{U}\left(X_{t}(\omega)\right) \mathrm{d} t=+\infty\right\}=1
$$

holds for all $x \in H$ and any open set $U \neq \emptyset$.

Proof. Let $U \neq \emptyset$ be arbitrary, by irreducibility $\varphi(U)>0$ and as $\varphi$ is Radon there exists a compact $M \subseteq U$ with $\varphi(M)>0$. By Lemma 5.1 the set $M$ is recurrent. Without any loss of generality we may assume that $\bar{U} \neq H$. Set $\eta \equiv \operatorname{dist}(M, H \backslash U)>$ 0 . By (A4) we can find $q>0$ such that

$$
\sup _{y \in M} \mathrm{P}_{y}\left\{\sup _{0 \leqslant t \leqslant q}\left\|X_{t}-y\right\| \geqslant \frac{\eta}{2}\right\}<\frac{1}{2} .
$$

Denote by $\sigma$ the first hitting time of $H \backslash U$; note that $\mathrm{P}_{x}\{\sigma<\infty\}=1$ for all $x \in H$ by Lemma 5.1 . Since

$$
\{\sigma>q\} \supseteq \bigcup_{y \in M}\left\{\sup _{0 \leqslant t \leqslant q}\left\|X_{t}-y\right\|<\frac{\eta}{2}\right\}
$$

we obtain

$$
\inf _{y \in M} \mathrm{P}_{y}\{\sigma>q\} \geqslant \frac{1}{2}
$$

Further, let $\tau$ be the first hitting time of $M$, for any $x \in H$ we have $\tau<\infty \mathrm{P}_{x}$-almost surely. Let us define by induction

$$
\begin{aligned}
\tau_{1} & =\tau, \\
\sigma_{n} & =\tau_{n}+\theta_{\tau_{n}} \sigma, \\
\tau_{n+1} & =\sigma_{n}+\theta_{\sigma_{n}} \tau,
\end{aligned}
$$

and set $Q_{n}=\left\{\omega ; \sigma_{n}(\omega)-\tau_{n}(\omega)>q\right\}$. For every $y \in H$ we obtain

$$
\begin{aligned}
\sum_{n=1}^{\infty} \mathrm{P}_{y}\left(Q_{n} \mid \mathscr{F}_{\tau_{n}}\right) & =\sum_{n=1}^{\infty} \mathrm{P}_{y}\left(\theta_{\tau_{n}}\{\sigma>q\} \mid \mathscr{F}_{\tau_{n}}\right) \\
& =\sum_{n=1}^{\infty} \mathrm{P}_{X\left(\tau_{n}\right)}\{\sigma>q\} \geqslant \sum_{n=1}^{\infty} \inf _{z \in M} \mathrm{P}_{z}\{\sigma>q\} \\
& \geqslant \sum_{n=1}^{\infty} \frac{1}{2}=+\infty \quad \mathrm{P}_{y} \text {-almost surely. }
\end{aligned}
$$


Therefore by the generalized Borel-Cantelli lemma

$$
\mathrm{P}_{y}\left(\limsup _{n \rightarrow \infty} Q_{n}\right)=1, \quad y \in H .
$$

Realizing that $\chi_{U}\left(X_{t}(\omega)\right)=1$ for $\tau_{n}(\omega) \leqslant t<\sigma_{n}(\omega)$ we see that (5.4) implies (5.3).

Note that under the assumptions of Proposition 5.4 the hypothesis (C) does hold, since the resolvent kernel $U$ is strong Feller by (A2), thus Lemma 5.2 implies that $\mu$ is Radon. Find an open set $V \neq \emptyset$ with $\mu(V)<\infty$, then (5.3) yields (C). Therefore, the statement (i) of Theorem 2.2 is proved.

Remark 5.3. As can be seen easily, if one replares the assumption (A3) in Proposition 5.4 with (E) then (5.3) holds for any open set $U \subseteq H$ such that $\varphi(U)>0$. This again yields $(\mathrm{C})$, since it suffices to find an open set $V$ with $\mu(V)<\infty$ and $\varphi(V)>0$. Note that there is a countable open cover $\left\{V_{n} ; n \in \mathbb{N}\right\}$ of $H$ with sets fulfilling $\mu\left(V_{n}\right)<\infty$, because $\mu$ is locally finite. Were $\varphi\left(V_{n}\right)=0$ for any $n$ then $\varphi(H)=0$, which would be a contradiction.

To proceed further we establish a ratio ergodic theorem, relying on the abstract Chacon-Ornstein result for Markov operators. First we quote several auxiliary results the proofs of which can be found in [37], §1.1.2. Define the measure $P_{\mu}$ on $\mathscr{F}$ by the formula (2.1), where $\mu$ is the $\sigma$-finite invariant measure provided by Lemma 5.3. Then $\mathrm{P}_{\mu}$ is a $\sigma$-finite $\left(\theta_{t}\right)$-invariant measure (that is. $\mathrm{P}_{\mu}\left(\theta_{t} B\right)=\mathrm{P}_{\mu}(B)$ for all $t>0$ and $B \in \mathscr{F})$. Let $\mathscr{J}$ stand for the $\sigma$-algebra of $\theta_{1}$-invariant sets, $\mathscr{J}=\{R \in \mathscr{\mathscr { F }}$; $\left.\mathrm{P}_{\mu}\left(\theta_{1} R \Delta R\right)=0\right\}, \Delta$ denoting the symmetric difference. Assume the equivalence of transition probabilities; if $B \in \mathscr{B}(H)$ is such that $P(1, x, B)=1$ for $\mu$-almost all $x \in B$ then either $\mu(B)=0$ or $\mu(H \backslash B)=0$. This yields that the measure $\mathrm{P}_{\mu}$ is trivial on $\mathscr{J}$, i.e. $\mathrm{P}_{\mu}(R)=0$ or $\mathrm{P}_{\mu}(\Omega \backslash R)=0$ for any $R \in \mathscr{J}$.

Proposition 5.5. Let the assumptions (A1), (E) and (C) be satisfied. Let $\mu$ be the $\sigma$-finite invariant measure for $X$. Let $\left(A_{t}\right),\left(B_{t}\right)$ be additive functionals, $\left(B_{t}\right)$ nonnegative, and suppose that $\mathrm{E}_{\mu}\left|A_{1}\right|<\infty, 0<\mathrm{E}_{\mu} B_{1}<\infty$. Then

$$
\mathrm{P}_{. r}\left\{\lim _{t \rightarrow \infty} \frac{A_{t}}{B_{t}}=\frac{\mathrm{E}_{\mu} A_{1}}{\mathrm{E}_{\mu} B_{1}}\right\}=1
$$

holds for all $x \in H$. Moreover,

$$
\lim _{t \rightarrow \infty} \frac{\mathrm{E}_{x} A_{t}}{\mathrm{E}_{x} B_{t}}=\frac{\mathrm{E}_{\mu} A_{1}}{\mathrm{E}_{\mu} B_{1}}
$$

holds for $\mu$-almost every $x \in H$. 
Proof. We apply the Chacon-Ornstein theorem in the same manner as it is done in [2], §II.2. First set

$$
M=\left\{\omega ; \sum_{i=0}^{\infty} \theta_{i} B_{1}(\omega)=+\infty\right\}
$$

and note that $M$ is obviously $\theta_{1}$-invariant, so either $\mathrm{P}_{\mu}(M)=0$, or $\mathrm{P}_{\mu}(\Omega \backslash M)=0$. But $\mathrm{E}_{\mu} B_{1}>0$ implies

$$
\mathrm{E}_{\mu}\left(\sum_{i=0}^{\infty} \theta_{i} B_{1}\right)=\sum_{i=0}^{\infty} \mathrm{E}_{\mu}\left(B_{1}\right)=\infty
$$

(the measure $P_{\mu}$ is $\theta_{1}$-invariant), so the former possibility is excluded.

Define a positive contraction $T: L^{1}\left(\mathrm{P}_{\mu}\right) \longrightarrow L^{1}\left(\mathrm{P}_{\mu}\right), u \longmapsto \theta_{1} u$. Let $V$ be the set whose existence is guaranteed by the assumption (C) and set $v=\int_{0}^{1} \chi_{V}\left(X_{s}\right) \mathrm{d} s$. Then $v \in L^{1}\left(\mathrm{P}_{\mu}\right)$ as

$$
\int_{\Omega} v \mathrm{dP}_{\mu}=\int_{0}^{1} \int_{H} P(s, y, V) \mathrm{d} \mu(y) \mathrm{d} s=\mu(V)<\infty,
$$

and we have

$$
\sum_{i=0}^{\infty} T^{i} v=\int_{0}^{\infty} \chi_{\bigvee^{\prime}}\left(X_{s}\right) \mathrm{d} s=\infty \quad \mathrm{P}_{\mu^{-}} \text {-almost everywhere. }
$$

This means that the operator $T$ is conservative (cf. [22], Theorem 3.1.6) and by the Chacon-Ornstein theorem and Neveu-Chacon identification theorem (see e.g. [22], Theorems 3.2.7 and 3.3.4) we obtain that

$$
\lim _{n \rightarrow \infty} \frac{\sum_{i=0}^{n} T^{i} A_{1}}{\sum_{i=0}^{n} T^{i} B_{1}}=\lim _{n \rightarrow \infty} \frac{A_{n}}{B_{n}}=\frac{\mathrm{E}_{\mu} A_{1}}{\mathrm{E}_{\mu} B_{1}}
$$

$\mathrm{P}_{\mu}$-almost everywhere. Now, exactly the same procedure as in [2] applies, hence we see that the discrete time result (5.5) implies that $\mathrm{P}_{\mu}(\Omega \backslash C)=0$, where

$$
C=\left\{\omega \in \Omega ; \lim _{t \rightarrow \infty} \frac{A_{t}}{B_{t}}=\frac{\mathrm{E}_{\mu} A_{1}}{\mathrm{E}_{\mu} B_{1}}\right\} .
$$

So there exists $N \in \mathscr{B}(H), \mu(N)=0$ and $\mathrm{P}_{x}(C)=1$ for all $x \notin N$. Let $y \in H$ be arbitrary, then

$$
\begin{aligned}
\mathrm{P}_{y}(C) & =\mathrm{P}_{y}\left(\theta_{1} C\right)=\mathrm{E}_{y} \mathrm{P}_{y}\left(\theta_{1} C \mid \mathscr{F}_{1}\right)=\mathrm{E}_{y} \mathrm{P}_{X(1)}(C)=\int_{H} \mathrm{P}_{w}(C) P(1, y, \mathrm{~d} w) \\
& =\int_{H \backslash N} \mathrm{P}_{w}(C) P(1, y, \mathrm{~d} w)=1,
\end{aligned}
$$


as $P(1, y, N)=0$ by $(\mathrm{E})$. The first assertion of Proposition 5.5 follows. To prove the second assertion define a positive contraction

$$
S: L^{1}(\mu) \longrightarrow L^{1}(\mu), \quad f \longmapsto \int_{H} f(z) P(1, \cdot, \mathrm{d} z) .
$$

As above, let $V$ be the set from the assumption (C). Set $h=\int_{0}^{1} P(s, \cdot V) \mathrm{d} s$, then $h \in L^{1}(\mu)$ and

$$
\sum_{n=0}^{\infty} S^{n} h(x)=\int_{0}^{\infty} P(s, x, V) \mathrm{d} s=+\infty, \quad x \in H,
$$

so $S$ is conservative. Let $\mathscr{A}$ be the $\sigma$-algebra of $S$-alssorbing sets (cf. [22], Definition 3.1.7). The equivalence of transition probabilities of the process $X$ yields easily that $\mu$ is trivial on $\mathscr{A}$ (that is, $\mu(A)=0$ or $\mu(H \backslash A)=0$ for each $A \in \mathscr{A}$ ). Define

$$
f(x)=\mathrm{E}_{x} A_{1}, \quad g(x)=\mathrm{E}_{x} B_{1}, \quad x \in H,
$$

then $f, g \in L^{1}(\mu), g \geqslant 0$, hence by the Chacon-Ornstein theorem

$$
\begin{aligned}
\lim _{N \rightarrow \infty} \frac{\sum_{n=0}^{N} S^{n} f}{\sum_{n=0}^{N} S^{n} g}= & \frac{\int_{H} f \mathrm{~d} \mu}{\int_{H} g \mathrm{~d} \mu}=\frac{\mathrm{E}_{\mu} A_{1}}{\mathrm{E}_{\mu} B_{1}} \\
& \mu \text {-almost everywhere on }\left\{x ; \sum_{n=0}^{\infty} S^{n} g>0\right\} .
\end{aligned}
$$

Note that

$$
S f(x)=\int_{H} \mathrm{E}_{z} A_{1} P(1, x, \mathrm{~d} z)=\mathrm{E}_{x} \mathrm{E}_{X(1)} A_{1}=\mathrm{E}_{x}\left(\theta_{1} A_{1}\right) .
$$

We know that

$$
\mathrm{P}_{\mu}\left\{\omega ; \sum_{i=0}^{\infty} \theta_{i} B_{1}(\omega)<\infty\right\}=0,
$$

thus also

$$
\mathrm{P}_{x}\left\{\omega ; \sum_{i=0}^{\infty} \theta_{i} B_{1}(\omega)<\infty\right\}=0
$$

for $\mu$-almost all $x \in H$. Therefore

$$
\sum_{n=0}^{\infty} S^{n} g(x)=\sum_{n=0}^{\infty} \mathrm{E}_{x}\left(\theta_{i} B_{1}\right)=\infty
$$

for $\mu$-almost every $x \in H$, and (5.6) yields that

$$
\lim _{n \rightarrow \infty} \frac{\mathrm{E}_{x} A_{n}}{\mathrm{E}_{x} B_{n}}=\frac{\mathrm{E}_{\mu} A_{1}}{\mathrm{E}_{\mu} B_{1}}
$$

holds for $\mu$-almost any $x$. Again, the proof can be completed as in [2]. 
The ratio ergodic theorem has many immediate consequences.

Corollary 5.6. Let the assumptions of Proposition 5.5 be fulfilled. Then

a) The process $X$ is Harris recurrent, that is

$$
\mathrm{P}_{x}\left\{\int_{0}^{\infty} \chi_{A}\left(X_{s}\right) \mathrm{d} s=+\infty\right\}=1
$$

holds for any $x \in H$ and all $A \in \mathscr{B}(H)$ such that $\mu(A)>0$.

b) Let $\mu(H)=\infty$. Then

$$
\lim _{T \rightarrow \infty} \frac{1}{T} \int_{0}^{T} \beta\left(X_{s}\right) \mathrm{d} s=0 \quad \mathrm{P}_{x} \text {-almost surely }
$$

for any $x \in H$ and $\beta \in L^{1}(\mu)$.

c) Let $\mu(H)<\infty$. Set $\mu^{*}(\cdot)=\mu(\cdot) / \mu(H)$, then

$$
\lim _{T \rightarrow \infty} \frac{1}{T} \int_{0}^{T} \psi\left(X_{s}\right) \mathrm{d} s=\int_{H} \psi \mathrm{d} \mu^{*} \quad \mathrm{P}_{x} \text {-almost surely }
$$

for any $x \in H$ and $\psi \in L^{1}(\mu)$.

Proof. a) Assume that one can find $z \in H$ and $A \in \mathscr{B}(H)$ with $\mu(A)>0$ such that

$$
\int_{0}^{\infty} \chi_{A}\left(X_{s}\right) \mathrm{d} s<\infty \quad \text { on a set } \Xi,
$$

where $P_{z}(\Xi)>0$. Let $V$ be the set introduced in the assumption $(C)$, then

$$
\lim _{T \rightarrow \infty} \frac{\int_{0}^{T} \chi_{A}(X(s)) \mathrm{d} s}{\int_{0}^{T} \chi_{V}(X(s)) \mathrm{d} s}=0 \quad \mathrm{P}_{z} \text {-almost surely on } \Xi .
$$

According to the ratio ergodic theorem the limit should be $\mu(A) / \mu(V)>0$, and this contradiction proves the Harris recurrence.

b) Fix $\varepsilon>0, x \in H$ and $\beta \in L^{1}(\mu)$. Without loss of generality assume that $\beta \geqslant 0$. As $\mu(H)=+\infty$ we can find $M \in \mathscr{B}(H)$ such that $\mu(M)<\infty$ and

$$
\frac{\int_{H} \beta \mathrm{d} \mu}{\mu(M)}<\frac{\varepsilon}{2} .
$$

By Proposition 5.5 for $\mathrm{P}_{x}$-almost any $\omega \in \Omega$ there exists $T_{0}(\omega)<\infty$ such that

$$
\frac{\int_{0}^{T} \beta\left(X_{s}\right) \mathrm{d} s}{\int_{0}^{T} \chi_{M I}\left(X_{s}\right) \mathrm{d} s} \leqslant \frac{\int_{H} \beta \mathrm{d} \mu}{\mu(M)}+\frac{\varepsilon}{2} \leqslant \varepsilon
$$


for any $T \geqslant T_{0}(\omega)$. It remains to realize that

$$
\frac{\int_{0}^{T} \beta\left(X_{s}\right) \mathrm{d} s}{T} \leqslant \frac{\int_{0}^{T} \beta\left(X_{s}\right) \mathrm{d} . s}{\int_{0}^{T} \chi_{M}\left(X_{s}\right) \mathrm{d} s}
$$

holds for any $T \geqslant 0$.

The last assertion of Corollary 5.6 is obvious.

Before we state our next proposition let us define the tail $\sigma$-algebra $\mathscr{T}$ of the process $X$ by

$$
\bar{T}=\bigcap_{t \geqslant 0} \sigma\left\{X_{r} ; r \geqslant t\right\}
$$

We say that the $\sigma$-algebra $\mathscr{T}$ is trivial if each measure $\mathrm{P}_{x}, x \in H$, is trivial on $\mathscr{T}$, i.e. $\mathrm{P}_{x}(M)=0$ or $\mathrm{P}_{x}(M)=1$ for all $M \in \mathscr{T}$.

Proposition 5.7. Let the assumptions (A1), (E) and (C) be fulfilled. Then

$$
\lim _{t \rightarrow \infty}\left\|P_{t}^{*} \pi-P_{t}^{*} \varrho\right\|=0
$$

holds for any probability measures $\pi, \varrho$ on $\mathscr{B}(H)$.

P r o of. We can repeat the argument from [9], 1 educing the proof of (5.7) to an analogous result for discrete time Markov chains (see [19]). Formally, only locally compact space valued processes are considered in [9] but omitting this assumption does not affect the proof. On the other hand, under our assumptions the proof further simplifies, so we recall liere the main steps.

First, note that $\left(X_{n}, n \in \mathbb{N}\right)$ is a Markov chain with the transition probability kernel $P(1, \cdot, \cdot)$. As all the measures $P(1, x, \cdot)$ are equivalent the chain $\left(X_{n}\right)$ is aperiodic; we check that $\left(X_{n}\right)$ is Harris recurrent Take an arbitrary $B \in \mathscr{B}(H)$, $0<\mu(B)<\infty$, ( $\mu$ being the invariant measure for $X)$, and set

$$
R=\left\{\omega \notin \Omega, \sum_{n=1}^{\infty} \chi_{B}\left(X_{n}(\omega)\right)=+\infty\right\} .
$$

The set $R$ is $\theta_{1}$-invariant, so wnow that either $\mathrm{P}_{\mu}(R)=0$ or $\mathrm{P}_{\mu}(\Omega \backslash R)=0$. As we have already noted, $T: L^{1}\left(\mathrm{P}_{\mu}\right) \longrightarrow L^{1}\left(\mathrm{P}_{\mu}\right), u \longmapsto \theta_{1} u$ is a conservative positive contraction, and $\chi_{B}\left(X_{1}\right) \in L^{1}\left(P_{\mu}\right)$, hence

$$
\sum_{n=0}^{\infty} T^{n} \chi_{B}\left(X_{1}\right)=\sum_{n=1}^{\infty} \chi_{B}\left(X_{n}\right) \in\{0, \infty\} \quad \mathrm{P}_{\mu} \text {-almost surely. }
$$


If $\mathrm{P}_{\mu}(R)=0$ then

$$
0=\mathrm{E}_{\mu}\left(\sum_{n=1}^{\infty} \chi_{B}\left(X_{n}\right)\right)=\sum_{n=1}^{\infty} \int_{H} P(n, z, B) \mathrm{d} \mu(z)=\sum_{n=1}^{\infty} \mu(B)
$$

and this is a contradiction. Therefore $\mathrm{P}_{\mu}(\Omega \backslash R)=0$ and there exists $N \in \mathscr{B}(H)$, $\mu(N)=0$ and $\mathrm{P}_{y}(R)=1$ for all $y \in H \backslash N$. Furthermore, for $x \in H$ arbitrary we obtain

$$
\begin{aligned}
\mathrm{P}_{x}(R) & =\mathrm{E}_{x} \mathrm{P}_{x}\left(\theta_{1} R \mid \mathscr{F}_{1}\right)=\mathrm{E}_{x} \mathrm{P}_{X(1)}(R)=\int_{H} \mathrm{P}_{y}(R) P(1, x, \mathrm{~d} y) \\
& =\int_{H \backslash N} \mathrm{P}_{y}(R) P(1, x, \mathrm{~d} y) .
\end{aligned}
$$

Since $P(1, x, N)=0$ we have $\mathrm{P}_{x}(R)=1$ for all $x \in H$.

We see that the Markov chain $\left(X_{n}\right)$ is aperiodic and Harris recurrent so its tail $\sigma$-algebra is trivial due to Theorem 1 of [19]. Lemma 3 of the same paper then implies

$$
\lim _{n \rightarrow \infty}\|P(n, x, \cdot)-P(n, y, \cdot)\|=0
$$

for any $x, y \in H$. We aim at establishing the triviality of the tail $\sigma$-algebra $\mathscr{T}$ of the process $X$. Take $A \in \mathscr{T}$, then there exist $A_{t} \in \mathscr{F}_{t}$ such that $\theta_{t} A_{t}=A$ for all $t \geqslant 0$. So

$$
\mathrm{P}_{x}(A)=\mathrm{P}_{x}\left(\theta_{t} A_{t}\right)=\mathrm{E}_{x} \mathrm{P}_{x}\left(\theta_{t} A_{t} \mid \mathscr{F}_{t}\right)=\mathrm{E}_{x} \mathrm{P}_{X(t)}\left(A_{t}\right)=P\left(t, x, A_{t}\right)
$$

and (5.8) yields that $\mathrm{P}_{x}(A)=\mathrm{P}_{y}(A)$ for all $x, y \in H$. Note that $\theta_{s} A_{t+s}=A_{t}$ for all $s \geqslant 0$, so $A_{t} \in \mathscr{T}$ as well and the function $x \mapsto \mathrm{P}_{x}\left(A_{t}\right)$ is constant on $H$. Moreover,

$$
\mathrm{P}_{x}(A)=\mathrm{E}_{x} \mathrm{P}_{x}\left(\theta_{n} A_{n} \mid \mathscr{F}_{n}\right)=\mathrm{E}_{x} \mathrm{P}_{X(n)}\left(A_{n}\right)=\mathrm{P}_{x}\left(A_{n}\right)
$$

By the martingale convergence theorem

$$
\mathrm{P}_{x}(A)=\mathrm{P}_{X(n)}\left(A_{n}\right)=\mathrm{E}_{x}\left(A \mid \mathscr{F}_{n}\right) \stackrel{n \rightarrow \infty}{\longrightarrow} \chi_{A} \quad \mathrm{P}_{x} \text {-almost surely }
$$

and this implies that either $\mathrm{P}_{x}(A)=0$ for all $x \in H$, or $\mathrm{P}_{x}(A)=1$ for all $x$, as desired.

The proof that the triviality of $\mathscr{T}$ is equivalent to (5.7) is standard, see e.g. [9], Theorem II.4. 
Let us state some consequences of Proposition 5.T.

Corollary 5.8. Let the assumptions of Proposition 5.7 be fulfilled. Let $\mu$ be the $\sigma$-finite invariant measure for $X$.

a) Let $\mu(H)<\infty$. Set $\mu^{*}(\cdot)=\mu(\cdot) / \mu(H)$, then $\mu^{*}$ is an invariant probability measure and

$$
\lim _{t \rightarrow \infty}\left\|P_{t}^{*} \pi-\mu^{*}\right\|=0
$$

for every probability measure $\pi$ on $\mathscr{B}(H)$. In particular,

$$
\lim _{t \rightarrow \infty}\left\|P(t, x, \cdot)-\mu^{*}\right\|=0
$$

for any $x \in H$.

b) Let $\mu(H)=\infty$. Then

$$
\lim _{t \rightarrow \infty} P_{t} f(x)=0
$$

for any $f \in L^{1}(\mu) \cap \mathbf{B}(H)$ and all $x \in H$. In particulat.

$$
\lim _{t \rightarrow \infty} P(t, x, D)=0
$$

whenever $x \in H$ and $D \in \mathscr{B}(H)$ satisfies $\mu(D)<x$.

Pro of. Only (5.9) requires proof, but we can proced, with obvious modifications, as in the proof of Theorem 2.7 in [18].

Pro of of Proposition 2.4. In the above proofs we have used the assumption on the existence of a recurrent compact set only to establish that the condition (C) is fulfilled. We aim at proving that (C) holds uncler the assumptions of Proposition 2.4 as well, since this will yield the Harris recurrence of the process $X$, therefore also the existence of a recurrent compact, as the invariant measure is known to be Radon.

Let $\mu$ be the Radon measure subinvariant for the resolvent kernel, take an open set $V \ni x_{0}$ such that $\mu(V)<\propto$, we have to prove that

$$
\int_{0}^{\infty} \chi_{V}\left(X_{s}\right) \mathrm{d} s=+\infty \quad \mathrm{P}_{x} \text {-almost surely, } x \in H .
$$

We will proceed as in the proof of Proposition 5.4. Find an open set $C \ni x_{0}$ such that $\operatorname{dist}(\bar{C}, H \backslash V) \equiv \eta>0$. By (A5) there exist " $>0$ and a closed ball $M \subseteq C$ satisfying $x_{0} \in M$ and

$$
\sup _{y \in M} P_{y !}\left\{\sup _{0 \leqslant t \leqslant q}\left\|X_{t}-y\right\| \geqslant \frac{11}{2}\right\}<\frac{1}{2} .
$$


At the point $x_{0}$ there exists a base of recurrent sets, thus we can choose the set $M$ recurrent. Denote by $\tau$ the first hitting time of $M$, let $\sigma$ stand for the first hitting time of $H \backslash V$. Obviously, (5.10) yields

$$
\inf _{y \in M} \mathrm{P}_{y}\{\sigma>q\} \geqslant \frac{1}{2}
$$

Let us define by induction

$$
\begin{aligned}
\tau_{1} & =\tau, \\
\sigma_{n} & =\tau_{n}+\theta_{\tau_{n}} \sigma, \\
\tau_{n+1} & =\sigma_{n}+\theta_{\sigma_{n}} \tau,
\end{aligned}
$$

and set $Q_{n}=\left\{\omega ; \sigma_{n}(\omega)-\tau_{n}(\omega)>q\right\} \cap\left\{\omega ; \tau_{n}(\omega)<\infty\right\}$. Just as in the proof of Proposition 5.4 we get

$$
\sum_{n=1}^{\infty} \mathrm{P}_{y}\left(Q_{n} \mid \mathscr{F}_{\tau_{n}}\right) \geqslant \frac{1}{2} \sum_{n=1}^{\infty} \chi_{\left\{\tau_{n}<\infty\right\}} \quad \mathrm{P}_{y^{-}} \text {-almost surely, } y \in H .
$$

Set $\Xi=\left\{\omega ; \tau_{n}(\omega)<\infty, n \in \mathbb{N}\right\}$, then by the generalized Borel-Cantelli lemma we obtain

$$
\mathrm{P}_{y}\left(\Xi \Delta \limsup _{n \rightarrow \infty} Q_{n}\right)=0, \quad y \in H .
$$

On the other hand, if $\omega_{0} \notin \Xi$ then $\tau_{m+1}\left(\omega_{0}\right)=\infty$ for an $m \geqslant 1$, which is possible only if $\sigma_{m}\left(\omega_{0}\right)=\infty$ since the set $M$ is recurrent. Hence

$$
\int_{0}^{\infty} \chi_{V}\left(X_{s}\left(\omega_{0}\right)\right) \mathrm{d} s=+\infty
$$

which completes the proof.

Proof of Proposition 2.5. The assumption (C) is trivially fulfilled as we can set $V=H$.

The proof of Theorem 2.2 is still not complete since it remains to establish that the condition (2.2) or the condition (2.8) are sufficient for positive recurrence. To do so, we need another construction of the $\sigma$-finite invariant measure, using the embedded Markov chain technique in the same way as in [20], [21], or [25]. This construction simplifies a bit if (2.2) is assumed from the very beginning, but we decided to present here the general $\sigma$-finite case as it is of independent interest. 
Proposition 5.9. Assume that (A1), (A2) and (E) are fulfilled. Let there exist a recurrent compact set $K \subseteq H$. Then there exists a $\sigma$-finite invariant measure $b$ for the process $X$. For $\eta>0$ denote by $\mathfrak{n}_{\eta}$ the first hitting time of $K$ after $\eta$, that is

$$
\mathfrak{n}_{\eta l}=\inf \left\{t>\eta ; X_{t} \in I^{*}\right\} .
$$

Assume that there exists $\delta>0$ such that

$$
\sup _{x \in K} \mathrm{E}_{x} \mathfrak{n}_{\delta}<\infty
$$

Then $b(H)<\infty$.

Proof. Denote by $\tau$ the first hitting time of $K$, for brevity we set $\mathfrak{n}=\mathfrak{n}_{\delta}$; obviously $\mathfrak{n}=\delta+\theta_{\delta} \tau$. Define a sequence of stopping times

$$
\begin{aligned}
\mathfrak{n}_{1} & =\mathfrak{n}, \\
\mathfrak{n}_{k+1} & =\mathfrak{n}_{k}+\theta_{\mathfrak{n}_{k}} \mathfrak{n}, \quad k \geqslant 1 .
\end{aligned}
$$

As $K$ is a recurrent set, $\mathfrak{n}_{k}<\infty$ and $X\left(\mathfrak{n}_{k}\right) \in K \mathrm{P}_{x}$-almost surely for all $x$ and $k \geqslant 1$. By Theorem 3 of [23] we have that $\widetilde{X}=\left(X\left(\mathfrak{n}_{k}\right), k \geqslant 1\right)$ is a homogeneous Markov chain in $K$, denote by $Q$ its transition probability kernel,

$$
Q(x, A)=\mathrm{E}_{x} \chi_{A}(X(\mathfrak{n})), \quad x \in K, A \in \mathscr{B}(K) .
$$

We claim that $\tilde{X}$ is Feller, that is

$$
\int_{K} f(z) Q(\cdot, \mathrm{d} z) \in \mathscr{C}_{b}(K) \quad \text { for any } \quad f \in \mathscr{C}_{b}(K) .
$$

Take $f \in \mathscr{C}_{b}(K)$ arbitrary and note that $f$ can be extencled to a function $F \in \mathscr{C}_{b}(H)$. Set $\psi(y)=\mathrm{E}_{y} F\left(X_{\tau}\right)$, then $\psi \in \mathbf{B}(H)$ and we have

$$
\begin{aligned}
\int_{K} f(z) Q(x, \mathrm{~d} z) & =\mathrm{E}_{x} f\left(X_{\mathfrak{n}}\right)=\mathrm{E}_{x} F\left(X_{\mathfrak{n}}\right)=\mathrm{E}_{x}\left(\theta_{\delta} F\left(X_{\tau}\right)\right) \\
& =\mathrm{E}_{x} \mathrm{E}_{X(\delta)} F\left(X_{\tau}\right)=\mathrm{E}_{x} \psi(X(\delta)) .
\end{aligned}
$$

By (A2) the function $x \longmapsto \mathrm{E}_{x} \psi(X(\delta))$ is continuous on $H$, hence on $K$. Therefore $\widetilde{X}$ is a homogeneous Feller Markov process in a compact state space $K$ and, consequently, there exists an invariant probability measure $\pi$ for $\widetilde{X}$; obviously $\pi$ can be considered as a measure on $\mathscr{B}(H)$. Set

$$
b(A)=\mathrm{E}_{\pi} \int_{0}^{\mathfrak{n}} \chi_{A}\left(X_{u}\right) \mathrm{d} u, \quad A \in \mathscr{B}(H),
$$


then $b$ is a $\sigma$-additive measure on $\mathscr{B}(H)$. We have to prove that $b$ is invariant for the process $X$, but the procedure from [21], Theorem IV.4.1, applies without any change. For convenience of the reader we repeat the proof. Take $f \in \mathbf{B}(H), f \geqslant 0$, and $t>0$ arbitrary. We want to establish that

$$
\int_{H} P_{t} f \mathrm{~d} b=\int_{H} f \mathrm{~d} b
$$

First note that

$$
\mathrm{E}_{\pi} \int_{0}^{\mathfrak{n}} f\left(X_{t+s}\right) \mathrm{d} s=\mathrm{E}_{\pi} \int_{0}^{\mathfrak{n}} \mathrm{E}_{X(s)} f\left(X_{t}\right) \mathrm{d} s .
$$

Indeed,

$$
\begin{aligned}
\mathrm{E}_{x} \int_{0}^{\mathfrak{n}} f\left(X_{t+s}\right) \mathrm{d} s & =\int_{0}^{\infty} \mathrm{E}_{x} \chi_{\{s<\mathrm{n}\}} f\left(X_{t+s}\right) \mathrm{d} s \\
& =\int_{0}^{\infty} \mathrm{E}_{x} \mathrm{E}_{x}\left(\chi_{\{s<\mathrm{n}\}} f\left(X_{t+s}\right) \mid \mathscr{F}_{s}\right) \mathrm{d} s \\
& =\mathrm{E}_{x} \int_{0}^{\infty} \chi_{\{s<\mathrm{n}\}} \mathrm{E}_{x}\left(f\left(X_{t+s}\right) \mid \mathscr{F}_{s}\right) \mathrm{d} s \\
& =\mathrm{E}_{x} \int_{0}^{\mathfrak{n}} \mathrm{E}_{x}\left(\theta_{s} f\left(X_{t}\right) \mid \mathscr{F}_{s}\right) \mathrm{d} s
\end{aligned}
$$

holds for all $x$ and (5.13) follows by the Markov property. Further, (5.13) implies that

$$
\begin{aligned}
\int_{H} P_{t} f(x) \mathrm{d} b(x) & =\mathrm{E}_{\pi} \int_{0}^{\mathfrak{n}} P_{t} f\left(X_{u}\right) \mathrm{d} u=\mathrm{E}_{\pi} \int_{0}^{\mathfrak{n}} \mathrm{E}_{X(u)} f\left(X_{t}\right) \mathrm{d} u \\
& =\mathrm{E}_{\pi} \int_{0}^{\mathfrak{n}} f\left(X_{t+u}\right) \mathrm{d} u=\mathrm{E}_{\pi} \int_{t}^{t+\mathfrak{n}} f\left(X_{s}\right) \mathrm{d} s .
\end{aligned}
$$

Obviously,

$$
\mathrm{E}_{\pi} \int_{t}^{t+\mathfrak{n}} f\left(X_{s}\right) \mathrm{d} s=\mathrm{E}_{\pi} \int_{0}^{\mathfrak{n}} f\left(X_{s}\right) \mathrm{d} s+\mathrm{E}_{\pi} \int_{\mathfrak{n}}^{t+\mathfrak{n}} f\left(X_{s}\right) \mathrm{d} s-\mathrm{E}_{\pi} \int_{0}^{t} f\left(X_{s}\right) \mathrm{d} s .
$$

The invariantness of $\pi$ yields

$$
\begin{aligned}
\mathrm{E}_{\pi} \int_{\mathfrak{n}}^{t+\mathfrak{n}} f\left(X_{s}\right) \mathrm{d} s & =\mathrm{E}_{\pi}\left(\theta_{\mathfrak{n}} \int_{0}^{t} f\left(X_{s}\right) \mathrm{d} s\right)=\mathrm{E}_{\pi} \mathrm{E}_{X(\mathfrak{n})} \int_{0}^{t} f\left(X_{s}\right) \mathrm{d} s \\
& =\mathrm{E}_{\pi} \int_{0}^{t} f\left(X_{s}\right) \mathrm{d} s .
\end{aligned}
$$


We conclude that

$$
\int_{H} P_{t} f(x) \mathrm{d} b(x)=\mathrm{E}_{\pi} \int_{0}^{\mathfrak{n}} f\left(X_{s}\right) \mathrm{d} s
$$

and (5.12) follows.

If (5.11) is assumed then $b(H)=\mathrm{E}_{\pi} \mathfrak{n}<\infty$ and the proof is complete. In the opposite case it remains to prove that $b$ is $\sigma$-finite. By Lemma 5.1 we may assume that $\varphi(K)>0$. (Note that there exist compacts of positive measure since $\varphi$ is Radon.) Moreover, let us realize that $b(K)<\infty$, for we have

$$
\begin{aligned}
b(K) & =\mathrm{E}_{\pi} \int_{0}^{\mathrm{n}} \chi_{K}\left(X_{s}\right) \mathrm{d} s=\mathrm{E}_{\pi} \int_{0}^{\delta} \chi_{K}\left(X_{s}\right) \mathrm{d} s+\mathrm{E}_{\pi}\left(\theta_{\delta} \int_{0}^{\tau} \chi_{K}\left(X_{s}\right) \mathrm{d} s\right) \\
& \leqslant \delta+\mathrm{E}_{\pi} \mathrm{E}_{X(\delta)} \int_{0}^{\tau} \chi_{K}\left(X_{s}\right) \mathrm{d} s=\delta
\end{aligned}
$$

as $X_{s}(\omega) \notin K$ if $0 \leqslant s<\tau(\omega)$. Taking into account the equivalence of transition probabilities we see that (5.12) implies

$$
0<\int_{H} P(1, y, K) \mathrm{d} b(y)=b\left(K^{\prime}\right)<\infty,
$$

therefore $b$ is $\sigma$-finite and Radon by Lemma 5.2 .

Remark 5.4. Note that the statement from Remark 2.2 holds true. Indeed, this can be established by the procedure used above, the only step requiring a modification being the proof of the Feller property of the embedded Markov chain. Besides the assumptions of Proposition 5.9, let (A4) be satisfied as well. We have to prove that the function

$$
x \longmapsto \mathrm{E}_{x} f\left(X_{\mathrm{p}}\right)
$$

is continuous on $K$ for any $f \in \mathscr{C}_{b}(K)$. Set $\psi(y)=\mathrm{E}_{y} f\left(X_{\tau}\right), y \in H$, as above, then $\psi \in \mathbf{B}(H)$ and

$$
\mathrm{E}_{x} f\left(X_{\mathrm{p}}\right)=\mathrm{E}_{x} \psi\left(X_{\sigma}\right)
$$

Lemma 4.1 implies easily that the function E. $\psi(X(\sigma \wedge t))$ is continuous on $V$ for any $t>0$ (see e.g. [11], Theorem 13.1). Further, for any $x \in K$ we have

$$
\begin{aligned}
& \left|\mathrm{E}_{x} \psi(X(\sigma))-\mathrm{E}_{x} \psi(X(\sigma \wedge t))\right| \leqslant 2\|\psi\|_{\infty} \mathrm{P}_{x}\{\sigma>t\} \\
& \leqslant \frac{2\|\psi\|_{\infty}}{t} \sup _{y \in K} \mathrm{E}_{y} \sigma \leqslant \frac{2\|\psi\|_{\infty}}{t} \sup _{y \in K} \mathrm{E}_{y} \mathfrak{p} \stackrel{t \rightarrow \infty}{\longrightarrow} 0
\end{aligned}
$$

and the Feller property of the cmbedded chain follows. 


\section{References}

[1] J. Azéma, M. Kaplan-Duflo, D. Revuz: Récurrence fine des processus de Markov. Ann. Inst. H. Poincaré Probab. Statist. 2 (1966), 185-220.

[2] J. Azema, M. Kaplan-Duflo, D. Revuz: Mesure invariante sur les classes récurrentes des processus de Markov. Z. Wahrscheinlichkeitstheorie und Verw. Gebiete 8 (1967), $157-181$.

[3] J. Azéma, M. Duflo, D. Revuz: Mesure invariante des processus de Markov recurrents. Séminaire de Probabilités III. Lecture Notes in Math. 88, Springer-Verlag, Berlin, 1969, pp. 24-33.

[4] R. N. Bhattacharya: Criteria for recurrence and existence of invariant measures for multidimensional diffusions. Ann. Probab. 6 (1978), 541-553; Correction. Ibid. 8 (1980), 1194-1195.

[5] A. Chojnowska-Michalik, B. Gotdys: Existence, uniqueness and invariant measures for stochastic semilinear equations on Hilbert spaces. Probab. Theory Related Fields 102 (1995), 331-356.

[6] G. Da Prato, A. Debussche: Stochastic Cahn-Hilliard equation. Nonlinear Anal. 26 (1996), 241-263.

[7] G. Da Prato, J. Zabczyk: Stochastic equations in infinite dimensions. Cambridge University Press, Cambridge, 1992.

[8] C. Dellacherie, P.-A. Meyer: Probabilities and potential, Part A. North-Holland, Amsterdam, 1978.

[9] M. Duflo, D. Revuz: Propriétés asymptotiques de probabilités de transition des processus de Markov récurrents. Ann. Inst. H. Poincaré Probab. Statist. 5 (1969), 233-244.

[10] E. Б. Дынкин: Основания теории марковских процессов. ГИФМЛ, Москва, 1959.

[11] Е. Б. Дынкин: Марковские процессы. ГИФМЛ, Москва, 1963.

[12] F. Flandoli, B. Maslowski: Ergodicity of the 2-D Navier-Stokes equation under random perturbations. Comm. Math. Phys. 171 (1995), 119-141.

[13] S. R. Foguel: Limit theorems for Markov processes. Trans. Amer. Math. Soc. 121 (1966), 200-209.

[14] T. Funaki: Random motion of strings and related stochastic evolution equations. Nagoya Math. J. 89 (1983), 129-193.

[15] R. J. Gardner, W. F. Pfeffer: Borel measures. Handbook of set-theoretic topology. North-Holland, Amsterdam 1984, pp. 961-1043.

[16] D. Gqtarek, B. Gotdys: On weak solutions of stochastic equations in Hilbert spaces. Stochastics Stochastics Rep. 46 (1994), 41-51.

[17] R. K. Getoor: Transience and recurrence of Markov processes. Séminaire de Probabilités XIV - 1978/79. Lecture Notes in Math. 784, Springer-Verlag, Berlin, 1980, pp. 397-409.

[18] N. C. Jain: Some limit theorems for a general Markov process. Z. Wahrscheinlichkeitstheorie und Verw. Gebiete 6 (1966), 206-223.

[19] B. Jamison, S. Orey: Markov chains recurrent in the sense of Harris. Z. Wahrscheinlichkeitstheorie und Verw. Gebiete 8 (1967), 41-48.

[20] Р. З. Хасьминский: Эргодические свойства возвратных диффузионных процессов и стабилизация решений задачи Коши для параболических уравнений. Теор. Вероятност. и Применен. 5 (1960), 196-214.

[21] Р. 3. Хасьминский: Устойчивость систем дифференциальных уравнений при случайных возмущениях их параметров. Наука, Москва, 1969.

[22] U. Krengel: Ergodic theorems. Walter de Gruyter, Berlin-New York, 1985.

[23] G. Maruyama: On the strong Markov property. Mem. Fac. Sci. Kyushu Univ. Ser. A 13 (1959), 17-29. 
[24] G. Maruyama, H. Tanaka: Some properties of one-dimensional diffusion processes. Mem. Fac. Sci. Kyusyu Univ. Ser. A 11 (1957), 117-141.

[25] G. Maruyama, H. Tanaka: Ergodic property of $N$-dimensional recurrent Markov processes. Mem. Fac. Sci. Kyushu Univ. Ser. A 13 (1959), 157-172.

[26] B. Maslowski: On probability distributions of solutions of semilinear stochastic evolution equations. Stochastics Stochastics Rep. 45 (1993), 17-44.

[27] B. Maslowski: Uniqueness and stability of invariant measures for stochastic differential equations in Hilbert spaces. Stochastics Stochastics Rep. 28 (1989), 85-114.

[28] B. Maslowski, J. Seidler: Ergodic properties of recurrent solutions of stochastic evolution equations. Osaka J. Math. 31 (1994), 965-1003.

[29] S. P. Meyn, R. L. Tweedie: Generalized resolvents and Harris recurrence of Markov processes. Doeblin and modern probability. Contemporary Mathematics Vol. 149, AMS, Providence, 1993, pp. 227-250.

[30] S. P. Meyn, R. L. Tweedie: Continuous time processes and sampled chains. Adv. in Appl. Probab. 25 (1993), 487-517.

[31] C. Mueller: Coupling and invariant measures for the heat equation with noise. Ann. Probab. 21 (1993), 2189-2199.

[32] S. Peszat, J. Seidler: Maximal inequalities and space-time regularity of stochastic convolutions. Math. Bohem. To appear.

[33] S. Peszat, J. Zabczyk: Strong Feller property and irreducibility for diffusions on Hilbert spaces. Ann. Probab. 23 (1995), 157-172.

[34] M. Scheutzow: Qualitative behaviour of stochastic delay equations with a bounded memory. Stochastics 12 (1984), 41-80.

[35] J. Seidler: Da Prato-Zabczyk's maximal inequality revisited I.. Math. Bohem. 118 (1993), 67-106.

[36] A. N. Shiryayev: Probability. Springer-Verlag, New York-Berlin, 1984.

[37] A. B. Скороход: Асимптотические методы теории стохастических дифференциальных уравнений. Наукова Думка, Киев, 1987

[38] L. Stettner: On the existence and uniqueness of invariant measure for continuous time Markov processes. Leîschetz Center for Dynamical Systems Preprint \# 86-18, April 1986.

[39] L. Stettner: Remarks on ergodic conditions for Markov processes on Polish spaces. Bull. Polish Acad. Sci. Math. 42 (1994), 103-114.

[40] D. W. Stroock, S. R. S. Varadhan: Diffusion processes with continuous coefficients II. Comm. Pure Appl. Math. 22 (j965), 479-530.

[41] D. W. Stroock, S. R. S. Varadhan: Cn the support of diffusion processes with applications to the strong maximum principie. Proceedings Sixth Berkeley Symposium Math. Statist. Probab., Vol. III.. Univ. of California Press, Berkeley-Los Angeles, 1972, pp. 333-ऽ.5.

[42] J. Zabczyk: Structurai. properties and limit behaviour of linear stochastic systems in Hilbert spaces. Mathematical control theory. Banach Center Publications Vol. 14, PWN, Warsaw, 1985, pp. 591-609.

[43] J. Zabczyk: Symmetric solutions of semilinear stochastic equations. Stochastic partial differential equations and applications II (Trento, 1988). Lecture Notes in Math. 1390, Springer-Verlag, Berlin, 1989, pp. 237-256.

Author's address: Mathematical Institute, Academy of Sciences of the Czech Republic, Žitná 25, 11567 Praha 1, Czech Republic, e-mail: seidler@math.cas.cz. 